\title{
A TALE OF TWO HERBIG Ae STARS, MWC 275 AND AB AURIGAE: COMPREHENSIVE MODELS FOR SPECTRAL ENERGY DISTRIBUTION AND INTERFEROMETRY
}

\author{
A. Tannirkulam, ${ }^{1}$ J. D. Monnier, ${ }^{1}$ T. J. Harries, ${ }^{2}$ R. Millan-Gabet, ${ }^{3}$ Z. Zhu, ${ }^{1}$ E. Pedretti, ${ }^{4}$ M. Ireland, ${ }^{5}$ \\ P. Tuthill,${ }^{5}$ T. Ten Brummelaar,${ }^{6}$ H. McAlister, ${ }^{6}$ C. Farrington, ${ }^{6}$ P. J. Goldfinger, ${ }^{6}$ \\ J. Sturmann, ${ }^{6}$ L. Sturmann, ${ }^{6}$ And N. Turner ${ }^{6}$ \\ Received 2008 May 19; accepted 2008 August 8
}

\begin{abstract}
We present comprehensive models for the Herbig Ae stars MWC 275 and AB Aur that aim to explain their spectral energy distribution (from UV to millimeter) and long-baseline interferometry (from near-infrared to millimeter) simultaneously. Data from the literature, combined with new mid-infrared (MIR) interferometry from the Keck Segment Tilting Experiment, are modeled using an axisymmetric Monte Carlo radiative transfer code. Models in which most of the near-infrared (NIR) emission arises from a dust rim fail to fit the NIR spectral energy distribution (SED) and sub-milliarcsecond NIR CHARA interferometry. Following recent work, we include an additional gas emission component with similar size scale to the dust rim, inside the sublimation radius, to fit the NIR SED and long-baseline NIR interferometry on MWC 275 and AB Aur. In the absence of shielding of starlight by gas, we show that the gas-dust transition region in these YSOs will have to contain highly refractory dust, sublimating at $\sim 1850 \mathrm{~K}$. Despite having nearly identical structure in the thermal NIR, the outer disks of MWC 275 and AB Aur differ substantially. In contrast to the AB Aur disk, MWC 275 lacks small grains in the disk atmosphere capable of producing significant $10-20 \mu \mathrm{m}$ emission beyond $\sim 7 \mathrm{AU}$, forcing the outer regions into the "shadow" of the inner disk.
\end{abstract}

Subject headings: circumstellar matter — planetary systems: protoplanetary disks — radiative transfer — stars: pre-main-sequence — techniques: interferometric

Online material: color figure

\section{INTRODUCTION}

Herbig Ae (HAe) stars are pre-main-sequence stars of intermediate mass $\left(1.5-3 M_{\odot}\right)$. They exhibit a robust excess in emission over stellar photospheric values from near-infrared (NIR) to the millimeter wavelengths. This excess is now attributed to the passive reprocessing of stellar light by dust in the circumstellar environment (Tuthill et al. 2001; Natta et al. 2001; Dullemond et al. 2001). The geometry of the circumstellar environment of HAe stars has been actively debated in the astronomy community over the last two decades. Some of the early workers in this field (Hillenbrand et al. 1992) showed that the spectral energy distribution (SED) of HAe stars could be explained by emission from circumstellar matter in disklike geometry. Others (Miroshnichenko et al. 1997) argued that the emission could also arise from dust in a spherical geometry around the star, proving the inadequacy of SED modeling alone in uniquely fixing the geometry of the circumstellar matter. The first observational evidence in favor of a disk geometry came from millimeter interferometry in the form of asymmetries detected (Mannings \& Sargent 1997) in the millimeter images. Asymmetries in the NIR emission were also detected by the Palomar Test-Bed Interferometer (PTI; Eisner et al. 2003, 2004), settling the debate in support of a disk geometry for circumstellar material in Herbig Ae stars.

Most interferometric studies of HAe stars have relied on simple geometric models (Mannings \& Sargent 1997; Millan-Gabet

\footnotetext{
1 University of Michigan, Astronomy Department, 500 Church Street, 1017 Dennison Building, Ann Arbor, MI 48109-1042; atannirk@umich.edu.

${ }^{2}$ University of Exeter, School of Physics, Stocker Road, Exeter, EX4 4QL, $\mathrm{UK}$.

${ }^{3}$ Michelson Science Center, Pasadena, CA 91125.

4 University of St. Andrews, Scotland, UK.

5 School of Physics, Sydney University, NSW 2006, Australia.

6 CHARA, Georgia State University, Atlanta, GA 30302-3969.
}

et al. 1999, 2001; Eisner et al. 2003, 2004; Monnier et al. 2005) that explain the emission geometry of the system in only narrow wavelength ranges. This method, albeit extremely useful in elucidating some of the morphology details, is not adequate for exploring the interdependency in structure of the inner and outer parts of the disk. A number of studies (Dullemond et al. 2001; Dullemond \& Dominik 2004; van Boekel et al. 2005a) have shown that the structure of the inner disk at fractions of an AU scale clearly affects the structure of the outer disk. A complete understanding of the circumstellar disk structure in HAe stars therefore requires models that simultaneously explain the SED and interferometry over a large wavelength range. Such models have begun to appear in the literature only recently (Pontoppidan et al. 2007; Kraus et al. 2008).

In this paper, we develop comprehensive disk models to explain the SED and interferometry of the HAe stars MWC 275 and $\mathrm{AB}$ Aur. MWC 275 and $\mathrm{AB}$ Aur are prototype pre-main-sequence stars of similar ages and spectral type with extensive circumstellar disks. Due to the availability of photometric and interferometric data over a large wavelength range, MWC 275 and AB Aur are ideal candidates for testing disk models for young stellar objects (YSOs). The extent of their circumstellar-dust disks was first measured by Mannings \& Sargent (1997) to be several hundred AU using the Owens Valley Radio Observatory (OWRO). Natta et al. (2004) resolved the MWC 275 disk in the millimeter range and reported a de-convolved, projected dust-disk size of $300 \mathrm{AU} \times$ 180 AU. More recently, Isella et al. (2007) analyzed IRAM, SMA, and VLA continuum and ${ }^{12} \mathrm{CO},{ }^{13} \mathrm{CO}$, and ${ }^{18} \mathrm{CO}$ line data constraining the gas-disk radius to be $540 \mathrm{AU}$ with the gas in Keplerian rotation around the central star. Scattered light studies of MWC 275 (Grady et al. 2000) and AB Aur (Grady et al. 1999; Oppenheimer et al. 2008) show the presence of arcs and rings in the circumstellar disk. Corder et al. (2005) resolved the AB Aur CO disk radius to be $\sim 600 \mathrm{AU}$, finding strong evidence for Keplerian rotation 
for the bulk of the disk. Corder et al. (2005) and Lin et al. (2006) detected spiral arms in $\mathrm{CO}$ emission with radii of $\sim 150 \mathrm{AU}$, while Fukagawa et al. (2004) detected similar structure in Subaru $H$-band scattered light images. AB Aur also has substantial envelope material on scales larger then 600 AU (Grady et al. 1999; Semenov et al. 2005; Corder et al. 2005; Lin et al. 2006).

MIR emission probes giant planet formation region in circumstellar disks (Calvet et al. 1992; Chiang \& Goldreich 1997; Dullemond et al. 2001) with the emission arising from warm dust ( $T>150 \mathrm{~K}$ ). Meeus et al. (2001) and van Boekel et al. (2005b) used the $10 \mu \mathrm{m}$ MIR silicate emission feature from MWC 275 and $\mathrm{AB}$ Aur to show that dust grains in these systems had grown larger than the typical interstellar medium grain sizes. Mariñas et al. (2006) imaged AB Aur at $11.7 \mu \mathrm{m}$ and found the emission FWHM size to be $17 \pm 4 \mathrm{AU}$, consistent with the flared disk models of Dullemond \& Dominik (2004). In this paper, we present new $10 \mu \mathrm{m}$ measurements of AB Aur and MWC 275 with the Keck Segment Tilting Experiment (Monnier et al. 2004; described in our $\S 2$ ). In contrast to AB Aur, the MWC 275 disk is unresolved by the Segment Tilting Experiment (maximum baseline of $10 \mathrm{~m}$ ), requiring the VLT Interferometer (100 $\mathrm{m}$ baseline) to probe its MIR structure (Leinert et al. 2004). These observations suggest that MWC 275 disk differs considerably from AB Aur, and we present a detailed comparison of the two disk structures in the discussion $(\S 6)$.

Thermal NIR emission probes hot regions (typically the inner AU) of the disk with temperatures greater than $700 \mathrm{~K}$. The NIR disks of MWC 275 and AB Aur were first resolved with Infrared Optical Telescope Array (IOTA) by Millan-Gabet et al. (1999, 2001) and subsequently observed at higher resolution with PTI (Eisner et al. 2004), Keck Interferometer (Monnier et al. 2005), and the Center for High Anglular Resolution Astronomy (CHARA) interferometer array (Tannirkulam et al. 2008). In Tannirkulam et al. (2008, hereafter T08), we showed that inner-disk models in which the majority of the $K$-band emission arises in a dust rim (Dullemond et al. 2001; Isella \& Natta 2005; Tannirkulam et al. 2007) fail to fit the CHARA data at milliarcsecond resolution. We also demonstrated that the presence of additional NIR emission (presumably from hot gas) inside the dust destruction radius can help explain the CHARA data and the NIR SED. First calculations for the effects of gas on rim structure (Muzerolle et al. 2004) showed that for plausible disk parameters, the presence of gas does not modify dust-rim geometry significantly. Besides a poorly understood interferometric visibility profile, MWC 275 also displays as yet ill-understood NIR and MIR SED time variability (Sitko et al. 2008), which has been interpreted as variation of the inner disk structure. In $\S \S 4$ and 5 we present a detailed analysis of the NIR visibility and SED for MWC 275 and AB Aur, placing constraints on the wavelength dependence of the opacity source inside the dust destruction radius.

In this study, we focus on (1) explaining the inner-disk structure and discussing important open problems and (2) modeling the MIR emission morphology of the disks and the shape of the MIR spectrum. The paper is organized into seven sections, with $\S 2$ detailing the observations. Section 3 explains the disk model and the modeling strategy. Sections 4 and 5 analyze MWC 275 and AB Aur SED and visibilities in relation to the disk models. We present a discussion on our results and our conclusions in $\S \S 6$ and 7 , respectively.

\section{NEW OBSERVATIONS AND LITERATURE DATA}

The properties of the circumstellar disks in MWC 275 and AB Aur have been constrained using infrared and millimeter interferometry and SEDs. We include new NIR interferometry from the CHARA array, MIR interferometry from the Keck Segment Tilting Experiment, and millimeter interferometry from the literature in our analysis. Optical and NIR photometry obtained at the MDM Observatories, and mid- and far-infrared data from ISO are also included to constrain model SED. We describe the data in detail in the following paragraphs.

$K$-band (central wavelength of $2.13 \mu \mathrm{m}, \Delta \lambda 0.3 \mu \mathrm{m}$ ) interferometry on MWC 275 and AB Aur, first presented in T08, was obtained using the CHARA array with the two-beam CHARA "Classic" combiner at the back end (ten Brummelaar et al. 2005). The targets were observed with multiple baselines of CHARA at a variety of orientations in eight runs between 2004 June and 2007 June. The longest baseline observation was $325 \mathrm{~m}$ (resolution $^{7}$ of 0.67 mas) for MWC 275 and $320 \mathrm{~m}$ (resolution of 0.68 mas) for AB Aur. The data were reduced using standard CHARA reduction software (ten Brummelaar et al. 2005) and these results were cross-checked with an independent code developed at University of Michigan. HD 164031, HD 166295, and HD 156365, with uniform-disk (UD) diameters of $0.83 \pm 0.08$ mas, $1.274 \pm$ 0.018 mas, and $0.44 \pm 0.06$ mas (Merand et al. [2005] and getCal ${ }^{8}$ ), were used as calibrators for MWC 275. AB Aur visibilities were calibrated with HD 29645 (UD diameter $0.54 \pm 0.07$ mas, getCal) and HD 31233 (UD diameter $0.76 \pm 0.13$ mas, getCal). During the reduction procedure the flux ratios between the two interferometer telescopes were carefully monitored for the source and the calibrators. Data points having a flux ratio variation greater than $10 \%$ of the mean, indicating large changes in instrument alignment, were discarded. Three MWC 275 data points were removed by this criterion. The procedure was adopted to minimize calibration errors caused by differences in beam overlap in the source and calibrator. The relative visibility errors, which include calibration errors, statistics, and uncertainties in calibrator size, are at the $\sim 6 \%$ level, typical for CHARA Classic. In T08, we showed the reduced data briefly in the form of visibility interferometric-baseline plots. Here we present the complete observing logs with the $u v$ coverage (see Figs. 1 and 2) and the calibrated visibilities listed in Tables 1 and 2 . NIR interferometry from IOTA (Monnier et al. 2006), PTI (Eisner et al. 2003, 2004), and the Keck Interferometer (Monnier et al. 2005) have also been included to constrain the models.

MWC 275 and AB Aur were observed with the Keck Segment Tilting Experiment (Monnier et al. 2004; Weiner et al. 2006) to study their MIR emission morphology. Four subsets of Keck primary mirror segments were repointed and rephased so as to achieve four separate sparse aperture Fizeau interferometers, each with a separate pointing origin on the LWS detector (Jones $\&$ Puetter 1993). The details of the experiment and the data reduction procedure are provided in Monnier et al. (2004) and J. D. Monnier et al. (2008, in preparation). The calibrated data are listed in Table 3. In addition to the Keck Segment Tilting data, we also include MWC 275 MIR interferometry from the Mid-Infrared interferometric instrument for the Very Large Telescope Interferometer (VLTI MIDI; Leinert et al. 2004) in our analysis.

Millimeter interferometry from Mannings \& Sargent (1997), Natta et al. (2004), Semenov et al. (2005), Lin et al. (2006), and Isella et al. (2007) are used to constrain the circumstellar disk masses and disk sizes and surface density profile. In conjunction with the interferometry, the shape of the millimeter SED provides information on sizes of the dust grains in the bulk of the circumstellar disks.

\footnotetext{
Resolution is defined as $\lambda / 2 D$, where $\lambda$ is wavelength of observation and $D$ is the interferometer baseline length.

${ }^{8}$ See http://mscweb.ipac.caltech.edu/gcWeb/gcWeb.jsp.
} 


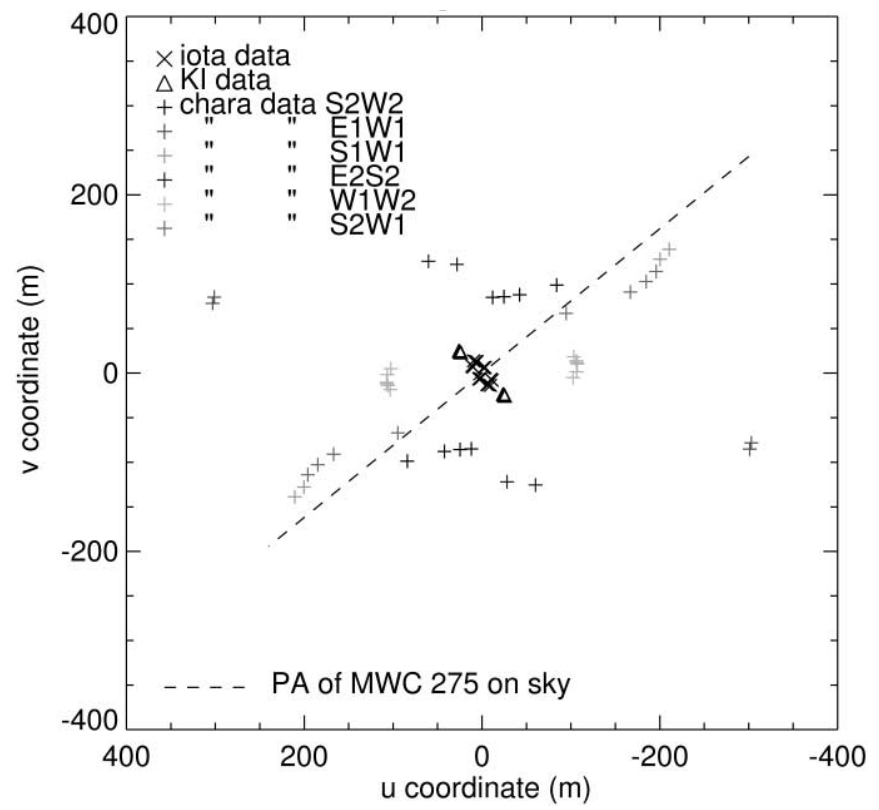

FIG. 1.-The $u v$ coverage for MWC 275. We include data from KI (Monnier et al. 2005), IOTA (Monnier et al. 2006), and CHARA (Tannirkulam et al. 2008 ) in our analysis. A position angle (measured east of north) of $136^{\circ}$ for MWC 275 is marked with a dashed line. [See the electronic edition of the Journal for a color version of this figure.]

To constrain the SED computed from radiative transfer models we obtained optical and NIR photometry on MWC 275 and AB Aur with the MDM $2.4 \mathrm{~m}$ telescope at Kitt Peak. Due to the high brightness of our targets, we defocussed the telescope during observations to avoid camera saturation. After bias correction, flat fielding, and background subtraction, the reduced images were used to obtain source counts using ATV, an aperture photometry code (Barth 2001). Targets were calibrated using Landoldt standards (Landolt 1983). The calibrated UBVRIJHK photometry are listed in Tables 10 and 11 in the Appendix. We also include photometry for a number of other YSOs that we observed. NIR photometry from Sitko et al. (2008), mid- and far-IR SED from Meeus et al. (2001), and millimeter fluxes (Mannings \& Sargent 1997; Natta et al. 2004; Semenov et al. 2005; Lin et al. 2006) were also used in the model analysis. Van Boekel et al. (2005b) modeled the $10 \mu \mathrm{m}$ spectra of a large sample of Herbig Ae stars and derived the mineralogy and typical grain sizes responsible for the emission. We use results from van Boekel et al. (2005b) for fixing the composition of dust grains in the atmosphere of the MWC 275 and AB Aur disks. A compilation of stellar properties and circumstellar disk properties extracted from the literature is listed in Tables 4, 5, 6 and 7.

\section{CIRCUMSTELLAR DISK MODELING}

The circumstellar material around MWC 275 and AB Aur is modeled as a passive dust disk reprocessing stellar radiation (Dullemond et al. 2001). The disk is in thermal and hydrostatic equilibrium and can be divided into three distinct regions (Fig. 3).

1. Curved Inner Rim.- Sufficiently close to the star (distance depends on stellar luminosity and dust sublimation temperatures), dust in the circumstellar disk reaches sublimation temperatures and evaporates. Inside of the evaporation radius, the disk is optically thin. The truncated dust disk is frontally illuminated by the star and forms a "rim" whose shape depends sensitively on dust properties (Isella \& Natta 2005; Tannirkulam et al. 2007). The rim puffs up, traps a significant fraction of stellar photons,

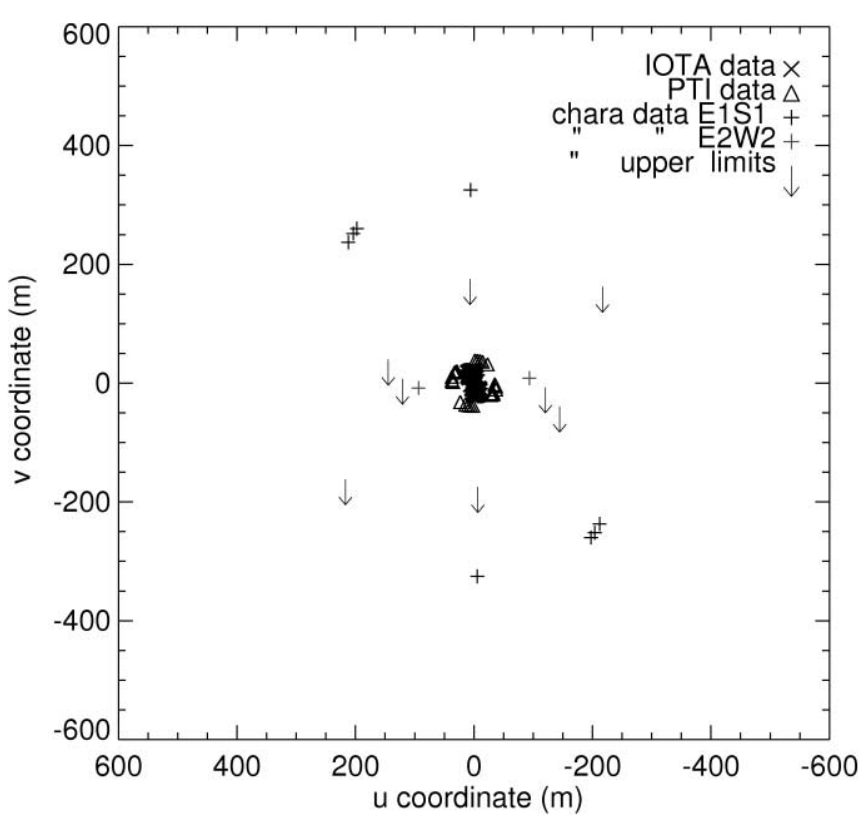

FIG. 2.- The $u v$ coverage for AB Aur. We include data from PTI (Eisner et al. 2004), IOTA, and CHARA ( Tannirkulam et al. 2008) in our analysis. [See the electronic edition of the Journal for a color version of this figure.]

and reradiates predominantly in the NIR (Dullemond et al. 2001).

2. Shadow Region.- The inner rim casts a geometric shadow on the region behind it (Dullemond et al. 2001; Dullemond \& Dominik 2004), preventing it from receiving direct starlight. The shadow is heated by scattered photons from the rim edge and through diffusion. The size of the shadow depends on the rim geometry, mass of dust in the outer disk, and dust grain properties in the outer disk.

3. Flared Disk.- If sufficient dust is present in the outer disk, the disk eventually emerges from the shadow and "flares." The flared disk emits radiation in the MIR and longer wavelengths.

Details of the modeling procedure and comparison to data are described below.

\subsection{The Monte Carlo Transfer Code: TORUS}

The calculations in this paper were performed using the TORUS Monte Carlo radiative-transfer code (Harries 2000; Harries et al. 2004; Kurosawa et al. 2004; Tannirkulam et al. 2007). Radiative equilibrium is computed using the Lucy (1999) algorithm on a two-dimensional, cylindrical adaptive-mesh grid. The initial density structure for the disk calculations is based on the canonical description of the $\alpha$-disk developed by Shakura \& Sunyaev (1973), viz.,

$$
\rho(r, z)=\rho_{0}\left(\frac{r}{r_{0}}\right)^{-\alpha} \exp \left[-\frac{1}{2} \frac{z^{2}}{h(r)^{2}}\right],
$$

where $r$ is the radial distance in the midplane, $r_{0}$ is some characteristic radius, $z$ is the distance perpendicular to the midplane, and $h(r)$ is the scale height, given by

$$
h(r)=h_{0}\left(\frac{r}{r_{0}}\right)^{\beta}
$$

with parameters of $\alpha=2.125$ and $\beta=1.125$, giving a radial dependence of the surface density of $\Sigma(r) \propto r^{-1.0}$. Once the 
TABLE 1

CHARA $u v$ Coverage and Visibility Data for MWC 275

\begin{tabular}{|c|c|c|c|c|c|}
\hline UT Date of Observation & $\begin{array}{c}u \\
(\mathrm{~m})\end{array}$ & $\begin{array}{c}v \\
(\mathrm{~m})\end{array}$ & Telescope Pair & Calibrated Visibility & Calibrator Names \\
\hline \multirow[t]{2}{*}{2004 Jul 9} & -210.61 & 138.79 & S1W1 & $0.150 \pm 0.008$ & HD 164031 \\
\hline & -200.38 & 127.78 & $\ldots$ & $0.143 \pm 0.009$ & $\ldots$ \\
\hline \multirow[t]{2}{*}{2005 Jul 22} & 106.91 & -11.88 & W1W2 & $0.218 \pm 0.011$ & HD 164031 \\
\hline & 103.22 & -18.38 & $\ldots$ & $0.227 \pm 0.009$ & $\ldots$ \\
\hline \multirow[t]{4}{*}{2005 Jul 26} & 102.45 & 5.03 & W1W2 & $0.260 \pm 0.014$ & HD 164031 \\
\hline & 106.45 & -1.61 & $\ldots$ & $0.241 \pm 0.011$ & $\ldots$ \\
\hline & 107.18 & -10.26 & $\ldots$ & $0.201 \pm 0.011$ & $\ldots$ \\
\hline & 105.94 & -13.95 & $\ldots$ & $0.232 \pm 0.011$ & ... \\
\hline \multirow[t]{3}{*}{ 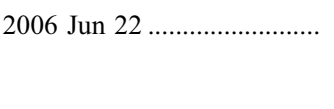 } & -11.99 & 84.94 & $\mathrm{~S} 2 \mathrm{~W} 2$ & $0.345 \pm 0.016$ & HD 164031 \\
\hline & -24.60 & 85.73 & $\ldots$ & $0.301 \pm 0.017$ & $\ldots$ \\
\hline & -42.30 & 87.77 & $\ldots$ & $0.203 \pm 0.013$ & $\ldots$ \\
\hline \multirow[t]{2}{*}{2006 Jun 23} & -301.23 & -85.20 & E1W1 & $0.0715 \pm 0.0043$ & HD 164031 \\
\hline & -302.93 & -78.34 & $\ldots$ & $0.0730 \pm 0.0044$ & $\ldots$ \\
\hline 2006 Jun 23 & -84.05 & 98.77 & S2W2 & $0.0925 \pm 0.0041$ & HD 164031 \\
\hline \multirow[t]{2}{*}{2006 Aug $23 \ldots \ldots \ldots$} & 60.15 & 125.48 & $\mathrm{E} 2 \mathrm{~S} 2$ & $0.181 \pm 0.010$ & HD 164031, HD 166295 \\
\hline & 28.16 & 121.90 & $\ldots$ & $0.189 \pm 0.011$ & $\ldots$ \\
\hline \multirow[t]{4}{*}{2007 Jun 17 .................................. } & -94.21 & 66.98 & S2W1 & $0.232 \pm 0.013$ & HD 164031, HD 156365 \\
\hline & -166.86 & 90.88 & $\ldots$ & $0.080 \pm 0.005$ & $\ldots$ \\
\hline & -184.56 & 102.73 & $\ldots$ & $0.096 \pm 0.006$ & $\ldots$ \\
\hline & -195.84 & 114.04 & $\ldots$ & $0.110 \pm 0.007$ & $\ldots$ \\
\hline
\end{tabular}

NoтE.-The array geometry is illustrated in Fig. 1, and ten Brummelaar et al. (2005).

temperature (we assume that the disk is in local thermodynamic equilibrium passively heated by the star, and gas and dust are thermally coupled) and dust sublimation (see next paragraph) structures have converged using the Lucy algorithm, the vertical disk structure is modified via the equation of hydrostatic equilibrium following a similar algorithm to that detailed by Walker et al. (2004). A self-consistent calculation for dust sublimation and disk temperature followed by a hydrostatic equilibrium calculation is repeated until the disk density structure has converged. Convergence is typically achieved in four iterations. Images and SEDs are subsequently calculated using a separate Monte Carlo algorithm based on the dust emissivities and opacities (Harries 2000).

The shape of the dust evaporation front is computed following Tannirkulam et al. (2007). The dust content is first reduced to a very low value in the computational grid for the circumstellar disk, to make each of the grid cells optically thin. Stellar photons then propagate through the disk and the temperature of grid cells is determined. Dust is added to cells that are cooler (see eq. [3] for sublimation temperature parameterization) than the sublimation temperature in small steps of $\tau$. The step size is a $\tau$ of $10^{-3}$ (computed at $5500 \AA$ ) for the first five dust growth steps. The step size is then increased logarithmically, first to $10^{-2}$, then to $10^{-1}$ and so on until a 100:1 gas to dust ratio is reached in each grid cell. The grid cell temperatures are recomputed after every dust growth step and the process is repeated until the shape of the dust sublimation region converges.

We use the Kurucz (1970) stellar atmosphere models as input spectra for the stars. We consider a mixture of $0.1,1.3$, and $50 \mu \mathrm{m}$ warm silicates (Ossenkopf et al. 1992) and power-law opacity millimeter grains (Mannings \& Sargent 1997; Natta et al. 2004) to model the opacity in the disk. The micron and submicron grain mixture is based on work by Meeus et al. (2001) and van Boekel et al. (2005b). To keep the analysis simple, the grain species are assumed to be well mixed with gas following a delta function size distribution. Dust scattering is not included in the models. Scattering does not seem to have significant impact on disk structure and interpretation of infrared interferometry for HAe stars (Dullemond \& Natta 2003; Pinte et al. 2008).

During the course of our modeling, and as outlined in T08, we realized that an additional emission component (Fig. 3), which we argue to be gas, is needed inside the dust destruction radius to explain the NIR SED and interferometry of MWC 275 and AB Aur (see $\S 4.1 .2$ for discussion on gas opacity). This additional component is not treated self-consistently in our modeling and is added after the dust-opacity-dominated circumstellar-disk model converges in structure. Calculations by Muzerolle et al. (2004) have shown that for parameters suitable to MWC 275 and AB Aur, gas does not significantly alter the structure of the dust rim, justifying our simple treatment for the NIR emission geometry.

TABLE 2

CHARA $u v$ Coverage and Visibility Data for AB Aur

\begin{tabular}{|c|c|c|c|c|c|}
\hline UT Date of Observation & $\begin{array}{c}u \\
(\mathrm{~m})\end{array}$ & $\begin{array}{c}v \\
(\mathrm{~m})\end{array}$ & Telescope Pair & Calibrated Visibility & Calibrator Names \\
\hline \multirow[t]{3}{*}{2006 Aug $23 \ldots \ldots \ldots \ldots \ldots \ldots$} & 212.04 & 237.05 & E1S1 & $0.095 \pm 0.005$ & HD 29645, HD 31233 \\
\hline & 203.79 & 251.87 & $\ldots$ & $0.120 \pm 0.006$ & .. \\
\hline & 197.53 & 259.95 & $\ldots$ & $0.123 \pm 0.007$ & $\ldots$ \\
\hline 2006 Dec 14 & -5.77 & -325.14 & E1S1 & $0.115 \pm 0.007$ & HD 29645, HD 31233 \\
\hline 2006 Dec 15 & -93.57 & 8.26 & E2W2 & $0.188 \pm 0.011$ & HD 29645, HD 31233 \\
\hline
\end{tabular}


TABLE 3

Keck Segment Tilting Experiment Baseline Coverage and $u v$ Averaged Visibility Data for MWC 275 and AB Aur

\begin{tabular}{cccc}
\hline \hline $\begin{array}{c}\text { UT Date } \\
\text { of Observation }\end{array}$ & $\begin{array}{c}\text { Baseline } \\
(\mathrm{m})\end{array}$ & $\begin{array}{c}\text { Calibrated } \\
\text { Visibility }\end{array}$ & $\begin{array}{c}\text { Calibrator } \\
\text { Names }\end{array}$ \\
\hline & MWC 275 & & \\
\hline 2004 Sep 1 ..................... & 3.03 & $0.969 \pm 0.049$ & v3879 Sgr \\
& 4.72 & $0.944 \pm 0.040$ & v3879 Sgr \\
& 5.49 & $0.946 \pm 0.036$ & v3879 Sgr \\
& 7.21 & $0.942 \pm 0.033$ & v3879 Sgr \\
& 8.43 & $0.963 \pm 0.033$ & v3879 Sgr \\
\hline & AB Aur & & \\
\hline 2004 Aug 30, 2004 Aug 31, & 3.03 & $0.870 \pm 0.039$ & Iota Aur \\
\& 2004 Sep 1 .................. & 4.72 & $0.823 \pm 0.027$ & Iota Aur \\
& 5.49 & $0.807 \pm 0.033$ & Iota Aur \\
& 7.21 & $0.753 \pm 0.047$ & Iota Aur \\
& 8.43 & $0.708 \pm 0.039$ & Iota Aur \\
& & & \\
\hline
\end{tabular}

In $\S \S 4.1 .1$ and 5.1, we demonstrate that the NIR emitting region in MWC 275 and $\mathrm{AB}$ Aur has a radius of $\sim 0.2 \mathrm{AU}$. Furthermore, long-baseline interferometry beyond the first visibility minimum constrains the gas and dust emission to be on similar length scales (Fig. 7). The two statements together imply that in the absence of shielding of the evaporation front by gas, the midplane sublimation temperature in the dust rim is $\sim 1850 \mathrm{~K}$ (see $\S 4.1 .1)$.

\subsection{Comprehensive Models for SED and Interferometry}

To fit the SED and visibilities of MWC 275 and AB Aur we adopted the following algorithm. First, we computed models for the dust evaporation front as described in $\S 3.1$. The inner edge of the dust disk is assumed to be dominated by grains larger than $1 \mu \mathrm{m}$ (Tuthill et al. 2001; Isella et al. 2006) and the evaporation front shape is set by the density dependence of dust sublimation

TABLE 4

Basic Stellar Properties and Photometry FOR MWC 275

\begin{tabular}{|c|c|}
\hline Property & Value \\
\hline 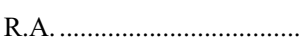 & $17^{\mathrm{h}} 56^{\mathrm{m}} 21.29^{\mathrm{s}}$ \\
\hline Decl. & $-21^{\circ} 57^{\prime} 21.8^{\prime \prime}$ \\
\hline Spectral Type ......................... & $\mathrm{A} 1 \mathrm{e}^{\mathrm{a}}$ \\
\hline$T_{\text {eff }}$ & $9500 \mathrm{~K}^{\mathrm{a}}$ \\
\hline 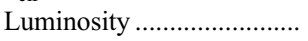 & $36 L_{\odot}$ \\
\hline Distance................................... & $122 \mathrm{pc}^{\mathrm{a}}$ \\
\hline 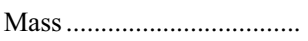 & $2.3 M_{\odot}^{\mathrm{a}}$ \\
\hline .......... & \\
\hline В & $6.98 \pm 0.08^{\mathrm{b}}$ \\
\hline$V$ & $6.84 \pm 0.06^{\mathrm{b}}$ \\
\hline$R$ & $6.86 \pm 0.05^{\mathrm{b}}$ \\
\hline I & $6.71 \pm 0.07^{\mathrm{b}}$ \\
\hline 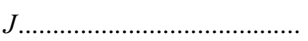 & $6.20 \pm 0.08^{\mathrm{b}}$ \\
\hline 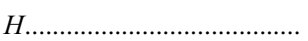 & $5.48 \pm 0.07^{\mathrm{b}}$ \\
\hline K) & $4.59 \pm 0.08^{\mathrm{b}}$ \\
\hline
\end{tabular}

${ }^{a}$ Stellar parameters from Monnier et al. (2006), Natta et al. (2004), and references therein.

${ }^{\mathrm{b}}$ Photometry obtained at MDM Observatories (longitude: $-111.67^{\circ}$, latitude: $31.95^{\circ}$ ) in 2006 June.
TABLE 5

Basic Stellar Properties and Photometry FOR AB Aur

\begin{tabular}{|c|c|}
\hline Property & Value \\
\hline Spectral Type ......... & $\mathrm{A} 0 \mathrm{pe}^{\mathrm{a}}$ \\
\hline$T_{\text {eff }}$ & $9772 \mathrm{~K}^{\mathrm{a}}$ \\
\hline 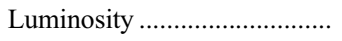 & $47 L_{\odot}^{\mathrm{a}}$ \\
\hline 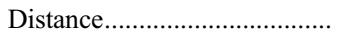 & $144 \mathrm{pc}^{\mathrm{a}}$ \\
\hline 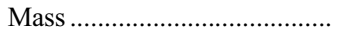 & $2.4 M_{\odot}^{\mathrm{a}}$ \\
\hline$U$ & $7.18 \pm 0.08^{b}$ \\
\hline$B$ & $7.14 \pm 0.04^{\mathrm{b}}$ \\
\hline$V$ & $7.01 \pm 0.04^{\mathrm{b}}$ \\
\hline$R$ & $6.96 \pm 0.05^{\mathrm{b}}$ \\
\hline I & $6.70 \pm 0.09^{\mathrm{b}}$ \\
\hline$J$ & $5.99 \pm 0.05^{\mathrm{b}}$ \\
\hline$H_{\ldots}$ & $5.28 \pm 0.05^{\mathrm{b}}$ \\
\hline K & $4.37 \pm 0.05^{\mathrm{b}}$ \\
\hline
\end{tabular}

temperatures (Isella \& Natta 2005; Tannirkulam et al. 2007). The $K$-band visibilities are computed for the model and compared with data. The normalization of the dust evaporation law is then adjusted so that the model visibilities fit the visibility data before the first visibility minimum. These models fail to fit the visibility beyond the minimum and do not have sufficient emission to explain the observed NIR SED. Therefore an additional emission component has been added inside the dust sublimation radius to reconcile the model with the visibility data and NIR SED.

With the inner-rim parameters fixed, we next focus on MIR and the millimeter disk. Millimeter interferometry results from the literature are used to fix disk masses and sizes. The majority of the dust mass is placed in millimeter-sized grains with a power-law opacity function (Natta et al. 2004). A small fraction ( $10 \%$ ) of the dust mass is in micron and submicron (small) grains with their relative mass fractions based on literature results (Meeus et al. 2001; van Boekel et al. 2005b). The physical extent of small grains is constrained with MIR imaging and interferometry. The model is then allowed to run to convergence. The model SED is

TABLE 6

MWC 275 Disk Properties from the Literature

\begin{tabular}{|c|c|}
\hline Property & Value \\
\hline \multicolumn{2}{|c|}{ Dust disk } \\
\hline Mass .......... & $0.0007 M_{\odot}^{\mathrm{a}}$ \\
\hline 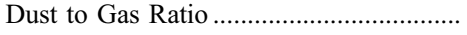 & 0.01 \\
\hline Surface Density Profile ................................. & $r^{-1 \mathrm{a}, \mathrm{b}}$ \\
\hline 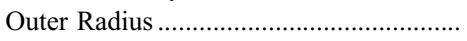 & $200 \mathrm{AU}^{\mathrm{b}}$ \\
\hline Inclination & $48^{\circ} \pm 2^{\circ \mathrm{c}}$ \\
\hline 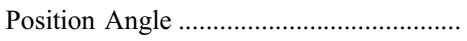 & $136^{\circ} \pm 2^{\circ \mathrm{c}}$ \\
\hline
\end{tabular}

Relative mass fractions of micron and submicron grains in the disk atmosphere ${ }^{\mathrm{d}}$

\begin{tabular}{|c|c|}
\hline $0.1 \mu \mathrm{m}$ Silicates. & $0.19_{-0.018}^{+0.009}$ \\
\hline $1.5 \mu \mathrm{m}$ Silicates. & $0.8_{-0.04}^{-0.010}$ \\
\hline PAH.. & $0.01_{-0.001}^{+0.001}$ \\
\hline
\end{tabular}

${ }^{a}$ Natta et al. (2004).

${ }^{b}$ Isella et al. (2007).

c This work and Wassell et al. (2006).

d van Boekel et al. (2005b). 
TABLE 7

AB Aur DISK PROPERTIES FROM THE LITERATURE

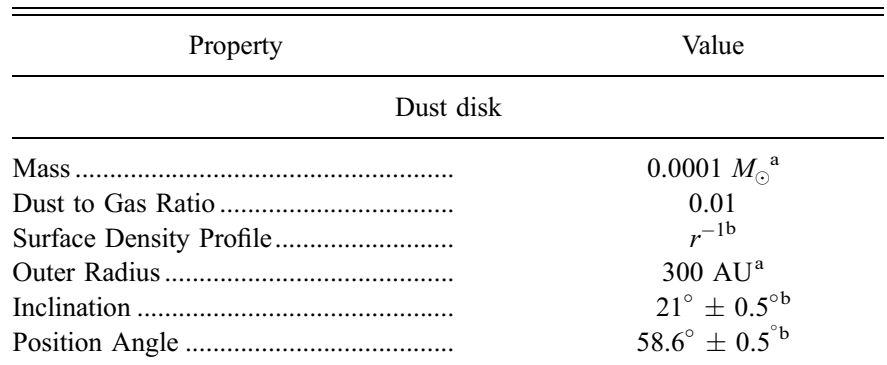

Relative mass fractions of micron and submicron grains in the disk atmosphere

\begin{tabular}{|c|c|}
\hline $0.1 \mu \mathrm{m}$ Silicates & $0.5_{-0.03}^{+0.03}$ \\
\hline $1.5 \mu \mathrm{m}$ Silicates ............ & $0.48_{-0.04}^{+0.03}$ \\
\hline 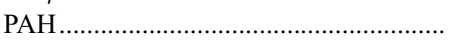 & $0.02_{-0.002}^{+0.001}$ \\
\hline
\end{tabular}

\footnotetext{
${ }^{\text {a }}$ Lin et al. (2006).

b Corder et al. (2005).

c van Boekel et al. (2005b).
}

compared with MIR and far-infrared data, the mass of the small grain population is then adjusted, and models are recomputed until a good fit to the MIR and far-infrared SED is obtained.

The MIR visibilities are computed for the SED-converged model and compared with the data and the spatial distribution of the small grain component is adjusted until model visibilities match with data. The only free parameters in our models are the absolute masses of the small grains, the masses of the $50 \mu \mathrm{m}$ silicate grains, and their spatial distribution. Each of the models is computationally expensive. To achieve fast convergence, the parameter space was varied by hand until a good fit was found for the observable quantities.

\section{MWC 275: ANALYSIS}

MWC 275 is a Herbig Ae star (refer to Table 4 for basic properties and photometry) with a total luminosity of $36 L_{\odot}$. The large stellar luminosity, coupled with the fact that the mass accretion rate is $\leq 10^{-7} M_{\odot} \mathrm{yr}^{-1}$ (Garcia Lopez et al. 2006) allows us to ignore accretion heating and model the MWC 275 circumstellar disk as a passive disk, reprocessing stellar radiation (Chiang \& Goldreich 1997; Dullemond et al. 2001). For our models, we choose the MWC 275 disk mass to be between 0.05 and $0.1 M_{\odot}$ (Natta et al. 2004) and a surface density profile that varies radially as $r^{-1}$ (Isella et al. 2007). The disk outer edge is truncated at $200 \mathrm{AU}$ and the bulk ( $\sim 80 \%)$ of the dust mass is assumed to reside in millimeter grains having an opacity with a wavelength dependence of $\lambda^{-1}$ at long wavelengths. Here, we describe in detail our modeling results for the NIR and MIR morphology of MWC 275.

\subsection{The Thermal NIR Disk}

\subsubsection{Visibilities}

Like many other Herbig Ae stars, MWC 275 shows a strong NIR excess over stellar photospheric values (Hillenbrand et al. 1992). This excess has been traditionally interpreted in terms of the dust disk being truncated by sublimation and forming a "rim." The rim intercepts stellar photons, reradiatiing predominantly in the NIR (Dullemond et al. 2001; Isella \& Natta 2005; Tannirkulam et al. 2007). However, in T08 we conclusively showed that models in which all of the NIR excess arises from dust rims alone cannot explain the CHARA interferometry data on MWC 275.
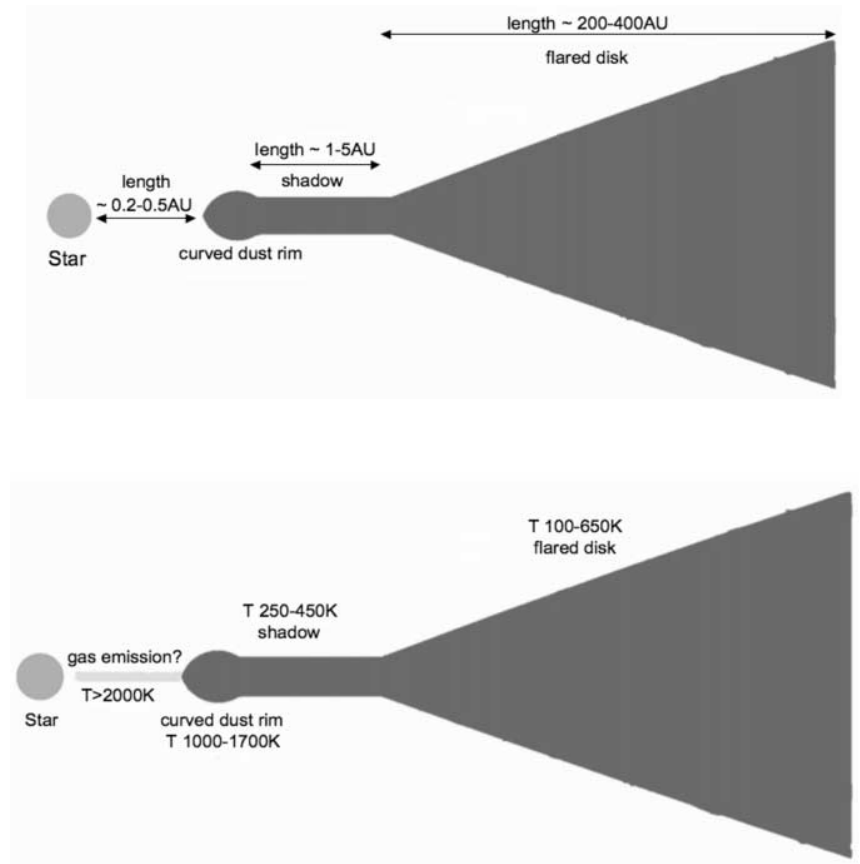

FIG. 3.- Schematic of disk models. Top: Flared disk with a curved inner rim. Bottom: An additional "smooth" emission component (presumably gas) has been added inside the dust destruction radius to explain MWC 275 and AB Aur NIR photometry and interferometry. Note that the models are not to scale. [See the electronic edition of the Journal for a color version of this figure.]

Our arguments in T08 were necessarily brief. We present a more elaborate analysis in this section.

MWC 275 observations allow us to clearly detect the asymmetry of the MWC 275 disk (see Appendix A.2), as having inclination $=48^{\circ} \pm 2^{\circ}$ and position angle (P.A.) $=136^{\circ} \pm 2^{\circ}$, consistent with the inclination of $51_{-9}^{+11}$ degrees and P.A. of $139^{\circ} \pm 15^{\circ}$ determined in Wassell et al. (2006) and the inclination of $46^{\circ} \pm 4^{\circ}$ and P.A. of $128^{\circ} \pm 4^{\circ}$ determined in Isella et al. (2007). The complete visibility data along each of the baselines are presented in Figure 4. Following T08, we show the data in a concise manner in Figure 5 using the notion of an "effective baseline"

$$
B_{\text {eff }}=B_{\text {projected }} \sqrt{\cos ^{2}(\theta)+\cos ^{2}(\phi) \sin ^{2}(\theta)},
$$

where $\theta$ is the angle between the $u v$ vector for the observation and the major axis of the disk and $\phi$ is the inclination of the disk. Under the flat disk assumption, the effective baseline correctly accounts for the change in resolution due to the disk inclination and P.A. (the geometry of thick disks is represented only approximately with optical depth effects and three-dimensional [3D] geometry of thick disks not being taken into account), allowing us to plot the visibility measurements as a function of one coordinate, simplifying presentation and analysis.

We attempt to fit the visibilities with a curved inner-rim model (the "standard" model) where the rim curvature (variation in cylindrical radius between rim midplane and the atmosphere) is set by the density dependence of dust sublimation temperatures, taken from Pollack et al. (1994). In this model, silicate grains sublimate at a higher temperature compared to other grains and hence fix the rim location. The rim is assumed to be composed of $1.3 \mu \mathrm{m}$ grains, as larger grains do not affect the rim shape and location significantly (Isella \& Natta 2005), at the same time making numerical convergence slower due to strong back-warming effects (Isella \& Natta 2005; Tannirkulam et al. 2007). For silicate 

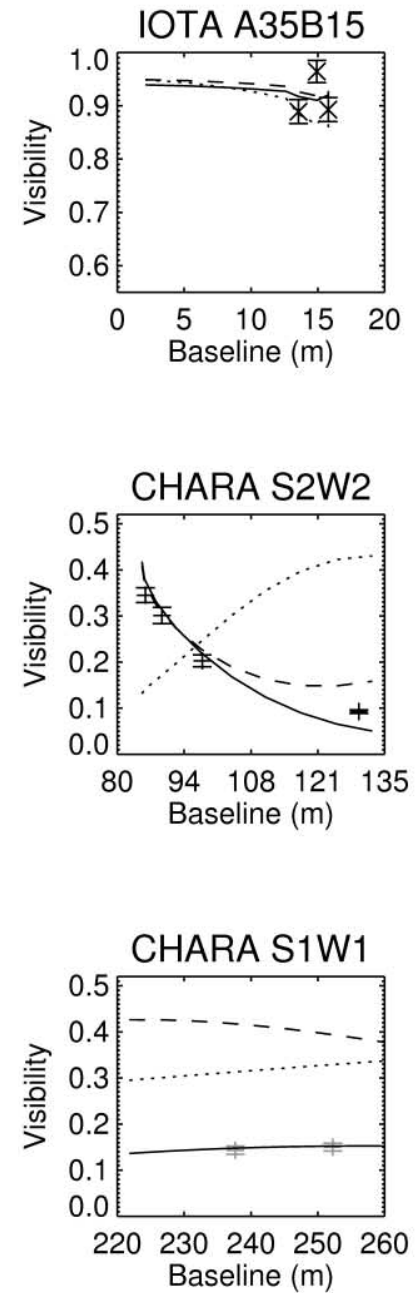
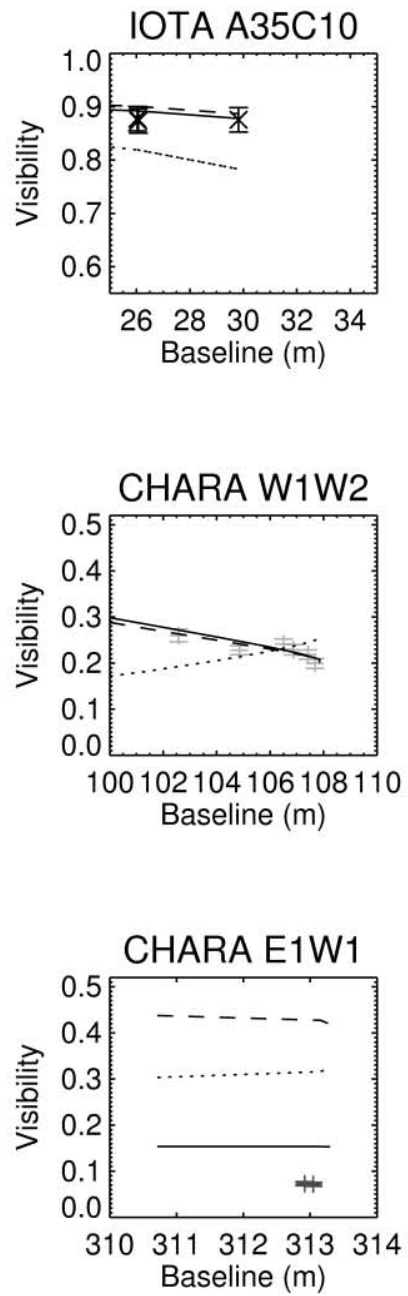
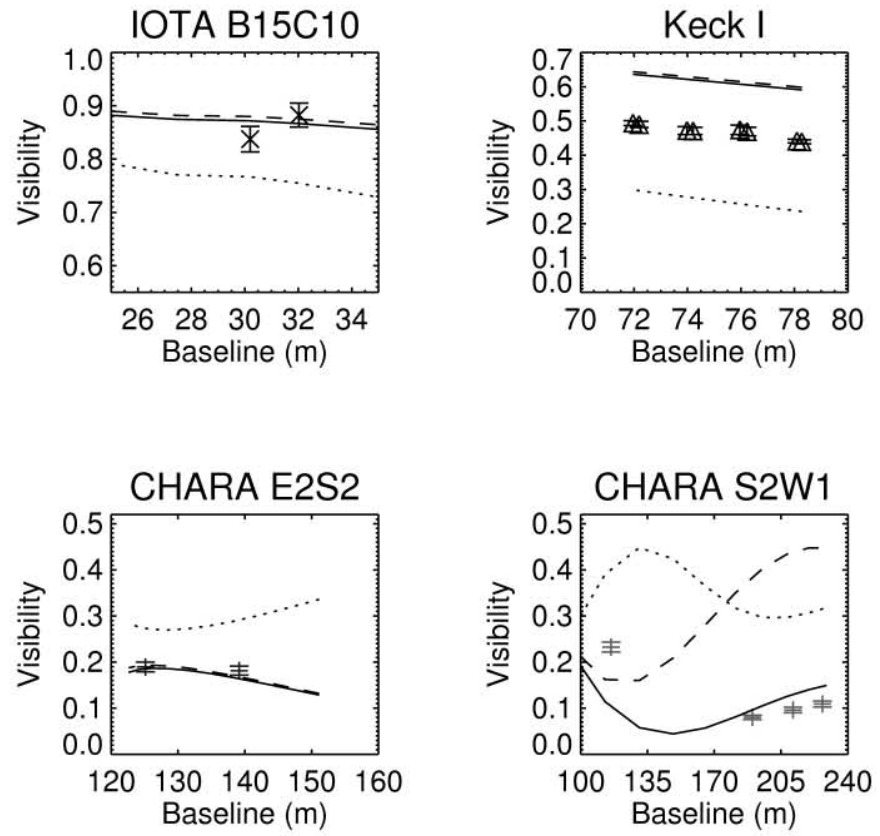

FIG. 4.- MWC 275 visibility data and model curves. The quoted model temperatures are at the base of the dust rim. The NIR size deduced from the Keck Inter-

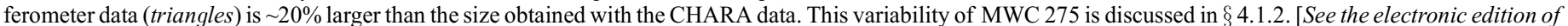
the Journal for a color version of this figure.]

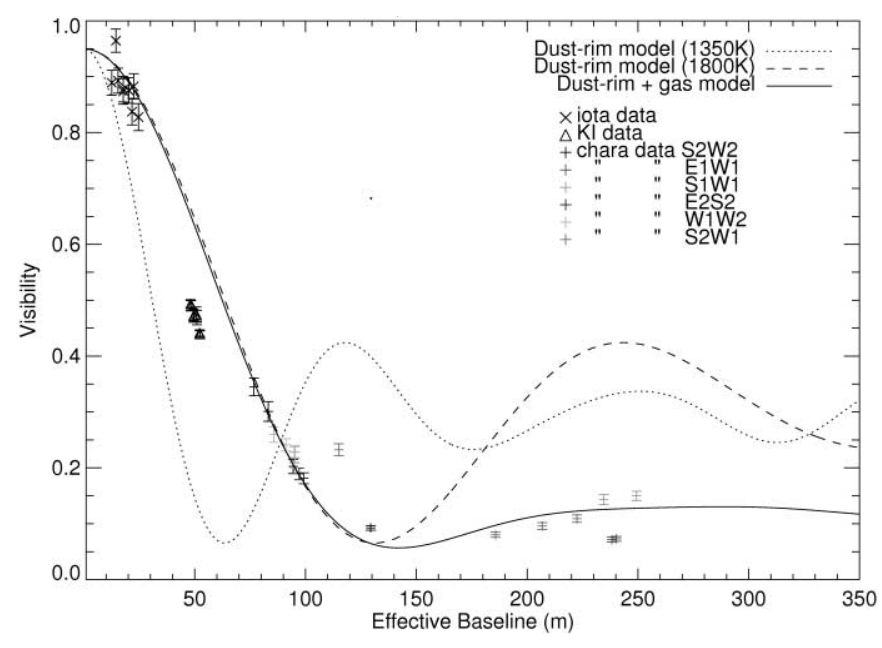

FIG. 5.-MWC 275 visibility vs. "effective baseline." Effective baselines are useful in presenting data along multiple $u v$ vectors in a concise manner (under the assumption of axial symmetry). The NIR size deduced from the Keck Interferometer data (triangles) is $\sim 20 \%$ larger than the size obtained with the CHARA data. This variability of MWC 275 is discussed in $\S 4.1 .2$. [See the electronic edition of the Journal for a color version of this figure.] dust, the evaporation temperature $T_{\text {evp }}$ can be parameterized as

$$
T_{\mathrm{evp}}=G\left[\frac{\rho_{\mathrm{gas}}(r, z)}{1 \mathrm{~g} \mathrm{~cm}^{-3}}\right]^{\gamma},
$$

where $G=2000 \mathrm{~K}, \gamma=1.95 \times 10^{-2}$, and $\rho_{\text {gas }}$ is the density of gas in $\mathrm{g} \mathrm{cm}^{-3}$ (see Isella \& Natta 2005, their eq. [16]). This parameterization, derived from a fit to sublimation temperatures recorded in the laboratory (Pollack et al. 1994), produces a dust rim with an inner edge at $0.36 \mathrm{AU}$ (Fig. 6, top left). The rim radius is too large to fit even the relatively short-baseline visibility data from IOTA (Figs. 4 and 5). In order to fit the data before the first visibility minimum, we had to increase the $T_{\text {evp }}$ normalization G by $\sim 30 \%$ from 2000 to $2600 \mathrm{~K}$. This increases the sublimation temperature at the base of the rim from $\sim 1350$ to $\sim 1800 \mathrm{~K}$. Figure 6 (top right) shows the synthetic $K$-band image for the rim with the increased normalization. The dashed line in Figure 4 traces the visibility for this model and provides a good fit to the shortbaseline $(<100 \mathrm{~m})$ data.

However, as seen in Figures 4 and 5, rim models which are sharply truncated due to dust sublimation and produce all of the NIR excess fail to fit observations beyond the first visibility minimum. These models display bounces in visibility at long baselines 

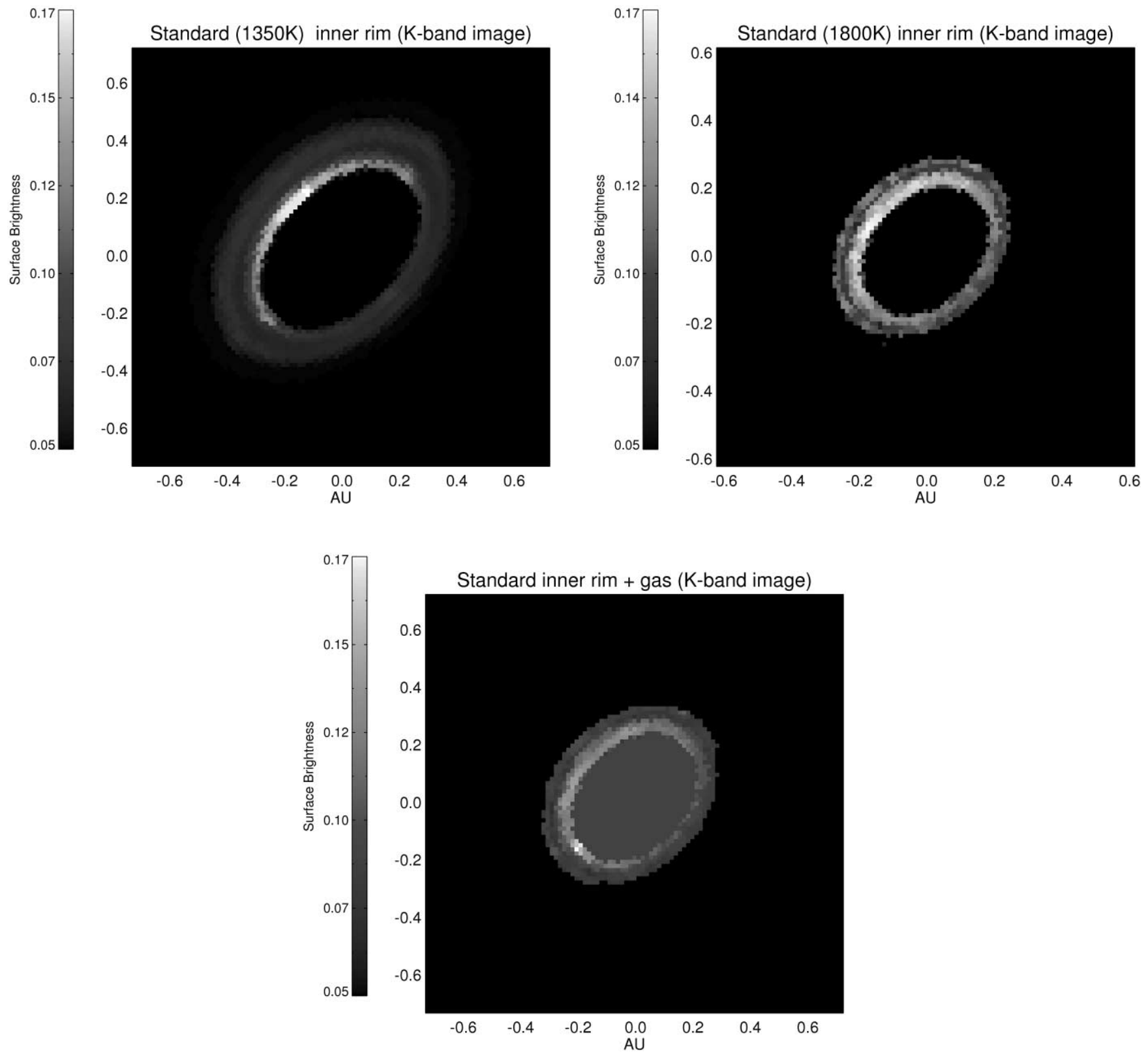

FIG. 6.-Inclined-disk models for NIR emission in MWC 275. The disk has an inclination of $48^{\circ}$ and a P.A. of $136^{\circ}$ (north is toward the top and east is on the left). The sense of the inclination is from Grady et al. (1999). Top left: Standard curved dust-rim-only model with rim-base temperature $\sim 1350 \mathrm{~K}$. Top right: Standard curved dust-rim-only model with rim-base temperature $\sim 1800 \mathrm{~K}$. Bottom: Curved dust-rim model with gas emission (modeled as a uniform disk centered on the star) inside the dust rim to smooth out the emission profile. [See the electronic edition of the Journal for a color version of this figure.]

(not seen in the data) because of the presence of sharp ringlike features with high spatial frequency components in the corresponding images, even for the smoothest rims physically plausible. In T08, we showed that the presence of a smooth emission component inside the dust destruction radius (Fig. 6, bottom) providing $56 \%$ of the total $K$-band emission helps fit the data well (solid line in Figs. 4 and 5). The NIR visibility data cannot constrain the surface brightness profile of the smooth emission component (we have adopted a constant surface brightness profile, a uniform disk for simplicity), but can constrain the size scale of the emission fairly robustly. Figure 7 shows a series of visibility curves where the smooth emission component is fixed to be $56 \%$ of the total emission and the radius of the uniform disk component is decreased by $15 \%$ successively from the initial radius of $0.23 \mathrm{AU}$ radius. The model image is then rescaled to maintain a good visibility fit at baselines shorter than $100 \mathrm{~m}$. It can be seen in Figure 7 that for uniform disk (UD) radii smaller than 0.19 AU, the model visibilities begin to deviate significantly from the observations. Thus, the CHARA data constrains the smooth emission component to be on the same length scale as the dust sublimation rim filling the region between the disk and the central star.

\subsubsection{SED}

Figure 8 shows the NIR SED for MWC 275. Besides failing to explain the NIR interferometry, the standard model also fails at producing sufficient NIR emission to explain the MWC 275 SED even in its "low" state. In T08, we showed that binarity and source variability cannot account for the discrepancy between the standard model and data. We argued that the presence of smooth emission inside the dust destruction radius can help explain the NIR visibility and account for the "missing" NIR flux in standard 


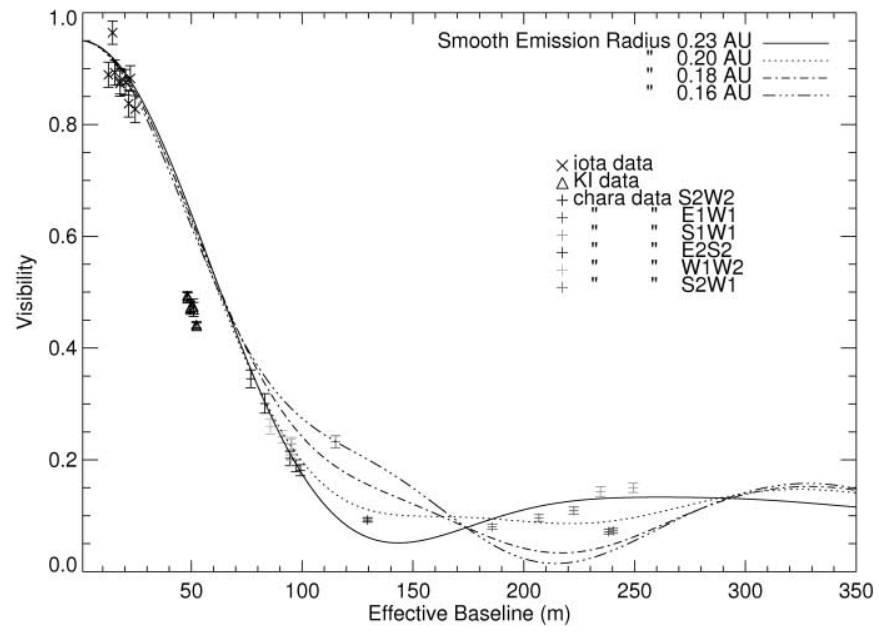

FIG. 7.-Constraining the size scale of the smooth emission component interior to the dust destruction radius in MWC 275. The model visibilities begin to deviate significantly from the data when the radius of the smooth emission component becomes smaller than 0.19 AU. [See the electronic edition of the Journal for a color version of this figure.]

models. Opacity candidates for the smooth emission component are (1) a dusty halo around the stars (Vinkovic et al. 2006a) and (2) gas inside the evaporation front. However, to fit the visibility data, the halo emission would have to be closer to the star than the dust destruction radius in the disk. This would require even higher dust-sublimation temperatures than the $\sim 1850 \mathrm{~K}$ we are adopting.

The most plausible physical mechanism for the smooth emission is hot gas. The required emission levels to explain the longbaseline $K$-band visibility data can be obtained with optically thin gas $(\tau \sim 0.15)$ with a temperature range of $2000-3000 \mathrm{~K}$ (Muzerolle et al. 2004; Eisner et al. 2007; Eisner 2007). Assuming that the gas has sufficient opacity to produce the difference in flux between the standard model and the observed photometry, we can place limits on the wavelength dependence of gas opacity. Figure 9 plots limits on the gas opacity (normalized at $K$ band) such that flux from the gas component + the standard model falls

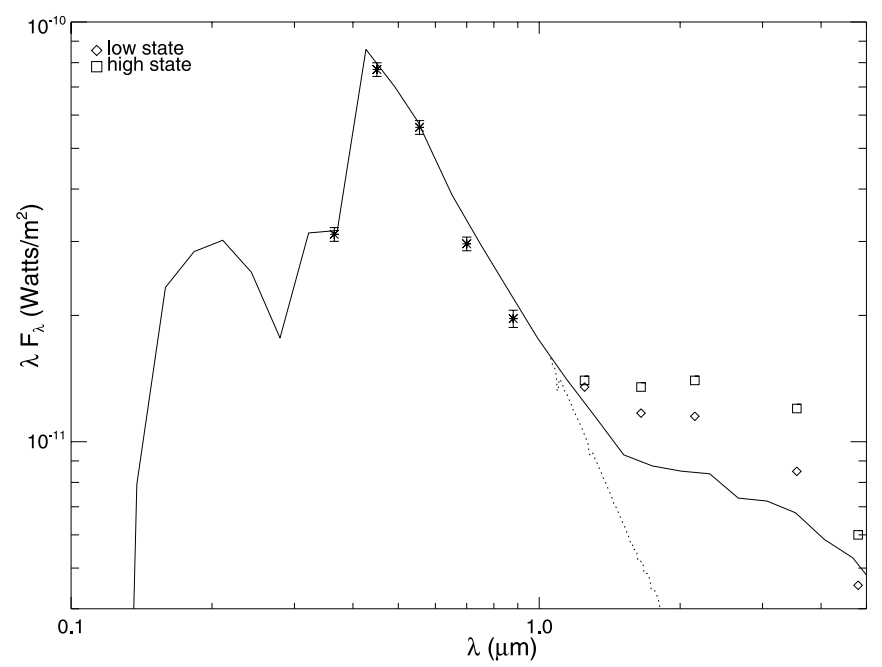

FIG. 8.-NIR SED for MWC 275. The asterisks are photometry points from MDM (Appendix, Table 4). The squares and diamonds are high- and lowstate measurements from Sitko et al. (2008). The solid line is the SED produced by the star + dust-rim-only model in the top right panel of Fig. 6. The dotted line is the SED of the star.
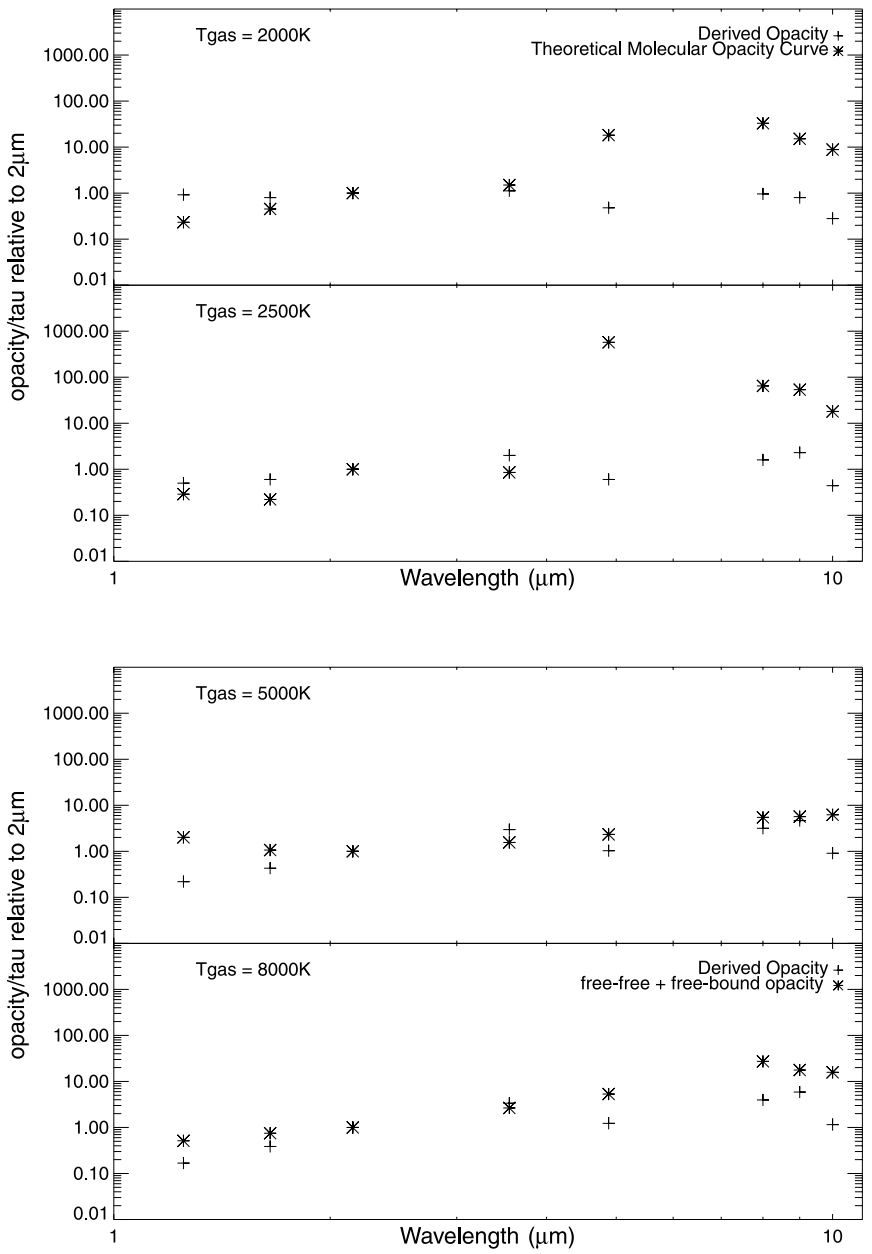

FIG. 9.-Plus signs: Empirically derived gas opacities from observed photometry and NIR disk models for MWC 275 (see Fig. 6). Top: Asterisks represent fiducial theoretical molecular absorption opacities smoothed over the photometry band for 2000 and $2500 \mathrm{~K}$ gas, respectively (Zhu et al. 2007). The opacity jump at $5 \mu \mathrm{m}$ is due to water vapor. Bottom: Gas absorption opacity at infrared wavelengths is dominated by free-free and free-bound transitions of $\mathrm{H}^{-}$at $5000 \mathrm{~K}$ and by hydrogen at $8000 \mathrm{~K}$ (Ferguson et al. 2005; Zhu et al. 2007).

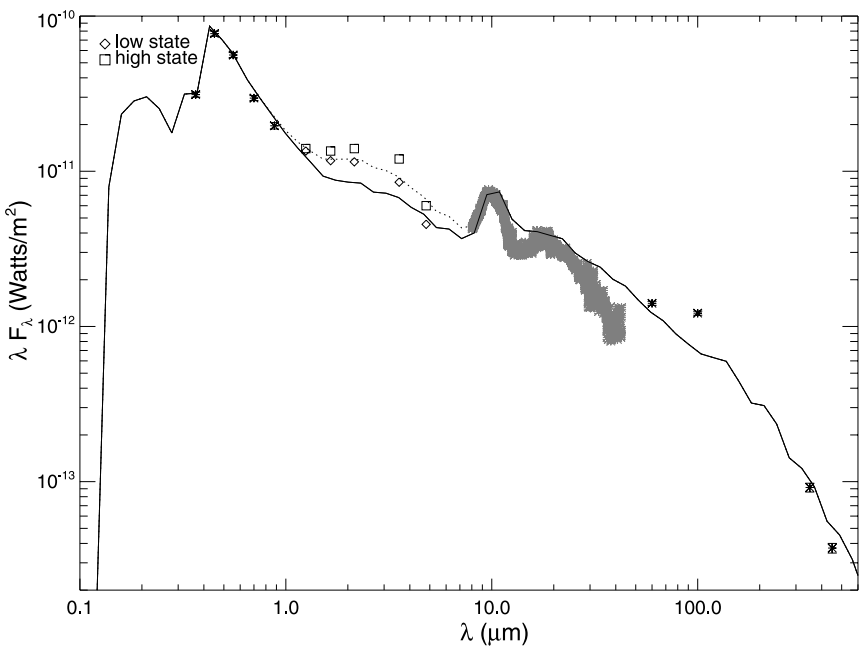

FIG. 10.-MWC 275 SED from UV to millimeter. The mid, far-infrared, and submillimeter data are from Meeus et al. (2001) and references therein. Solid line: Dust-disk model SED (see $\S 4.2$ ). Dotted line: Dust-disk + smooth-emission SED. The smooth component is modeled as optically thin gray emission at $2500 \mathrm{~K}$. The relative contributions of star, dust, and gas to the total integrated flux are $0.79,0.16$, and 0.05 , respectively. 

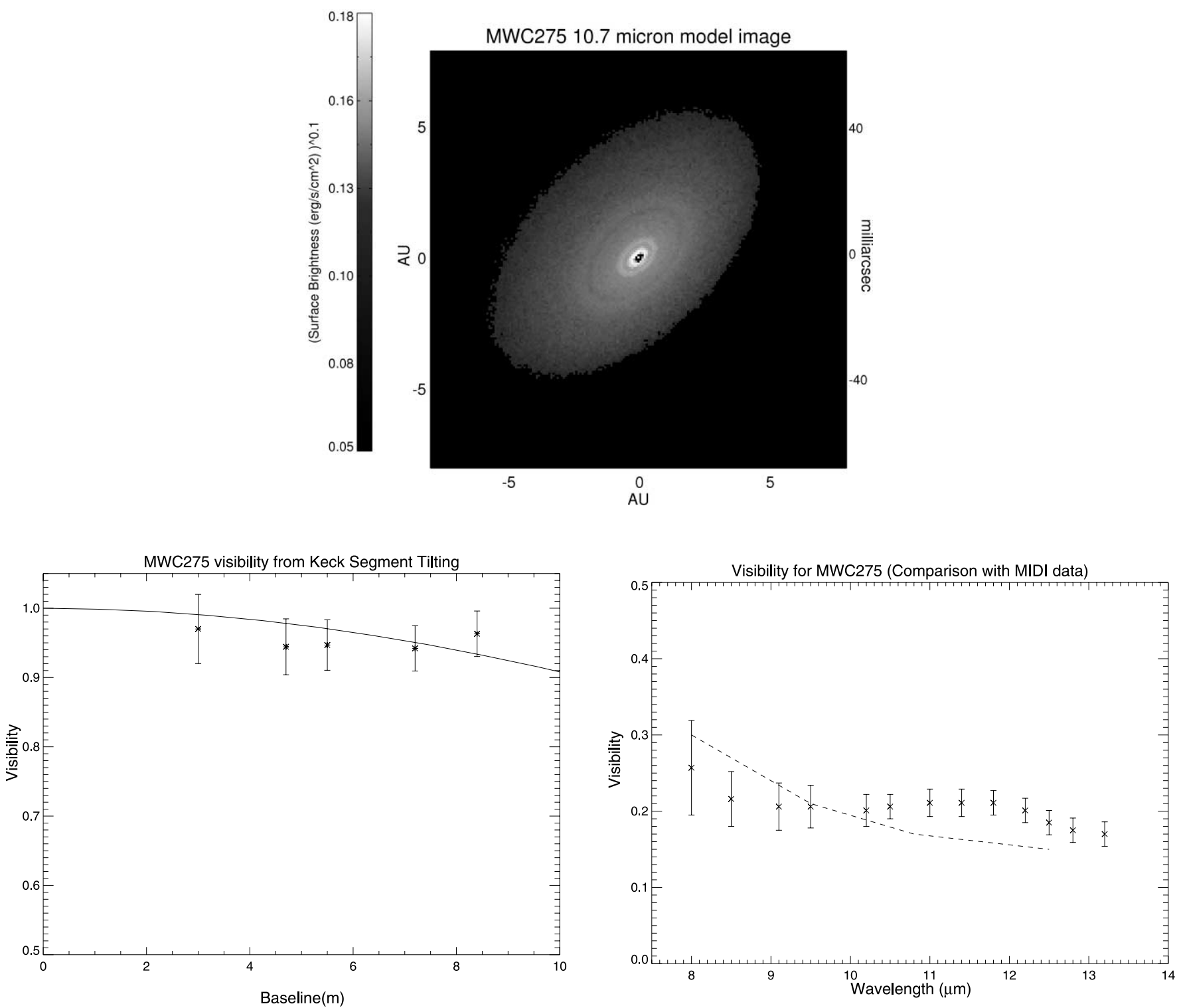

FIG. 11.-MIR image and visibilities for MWC 275. The disk has an inclination of $48^{\circ}$ and a P.A. of $136^{\circ}$ (north is toward the top and east is on the left). Top: Synthetic $11 \mu \mathrm{m}$ TORUS image. Bottom left: Azimuthally averaged $10.7 \mu \mathrm{m}$ visibilities from the Keck Segment Tilting Experiment (Monnier et al. 2008). The "stars" are measured values and the solid line is the model visibility. MWC 275 is not resolved by Keck. Bottom right: Model visibilities compared with MIDI (Leinert et al. 2004) data. The MIDI data was obtained at a projected baseline of $\sim 99 \mathrm{~m}$ and a P.A. of $16^{\circ}$, nearly aligned with disk minor axis. [See the electronic edition of the Journal for a color version of this figure.]

within $10 \%$ of the observed photometry (we have assumed that gas does not significantly alter the geometry of the dust rim). In the next two paragraphs we compare theoretical gas-opacity curves with our empirically derived opacity from SED.

Figure 9 shows the wavelength dependence of molecular (Ferguson et al. 2005; Zhu et al. 2007) and free-free + free-bound (henceforth FF-BF) opacity, both good candidates for the gas emission (refer to T08). At $5000 \mathrm{~K}$, FF-BF opacity (Ferguson et al. 2005) agrees well with the derived opacity at long wavelengths but overshoots limits shortward of $2 \mu \mathrm{m}$. At temperatures greater than $8000 \mathrm{~K}$, FF-BF opacities rise quickly with wavelength, producing excessive MIR light.

Theoretical molecular opacities compare fairly well with the empirical derivation between 1 and $4 \mu \mathrm{m}$. Beyond $4 \mu \mathrm{m}$, theoretical molecular opacities rise rapidly with wavelength. However, the observed SED can be matched with models only if the gas opacity is flat between 4 and $9 \mu \mathrm{m}$. In addition, at 2000 and $2500 \mathrm{~K}$ strong opacity bands of $\mathrm{CO}$ and water vapor are present at 2.5 and $5 \mu \mathrm{m}$, respectively, which have not been observed in MWC 275 . This suggests that if molecules were contributing to the bulk of NIR opacity, then some of the species providing opacity between 4 and $8 \mu \mathrm{m}$ in Ferguson et al. (2005) and Zhu et al. (2007) are being destroyed in the vicinity of Herbig Ae star MWC 275. We note that FF-BF opacities seem to better fit the empirically derived values than molecular opacity.

Sitko et al. (2008) have obtained fairly dense time coverage on the NIR and MIR SED of MWC 275. The NIR SED shows variability at the $20 \%$ level. During the same period, the flux in the visible shows no detectable change, indicating that stellar luminosity remained fairly constant. Sitko et al. (2008) interpret their observations as variations in the structure of the thermal NIR disk. A variation in the NIR morphology of MWC 275 was also detected in the interferometry. The NIR disk size deduced from the Keck Interferometer data (2003 April epoch, Figs. 4 and 5) is $\sim 20 \%$ larger than the size obtained with the CHARA data (2004 June-2006 August epochs). The size determined from the S2W1 
TABLE 8

MWC 275 Model-disk Properties Constrained by This Work

\begin{tabular}{|c|c|}
\hline Property & Value \\
\hline \multicolumn{2}{|l|}{ Dust disk } \\
\hline 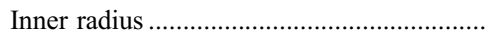 & $0.22 \mathrm{AU}^{\mathrm{a}}$ \\
\hline K-band flux contribution from dust rim..... & $29 \%{ }^{\mathrm{a}}$ \\
\hline Mass fractions of dust components............. & See Fig. 12 \\
\hline \multicolumn{2}{|c|}{ NIR gas disk } \\
\hline Surface brightness profile ............................. & Constant (poorly constrained) \\
\hline 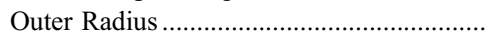 & $0.22 \mathrm{AU}^{\mathrm{a}}$ \\
\hline K-band flux contribution ... & $56 \%{ }^{\mathrm{a}}$ \\
\hline 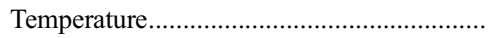 & $>1800 \mathrm{~K}$ \\
\hline 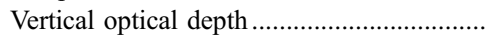 & $0.15^{\mathrm{a}}$ \\
\hline 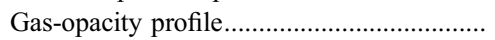 & See Fig. 9 \\
\hline
\end{tabular}

a Tannirkulam et al. (2008). The star contributes $10 \%$ of the K-band flux and an extended envelope (Monnier et al. 2006) contributes 5\%.

2007 June data also differs at the $\sim 25 \%$ level from the size obtained from earlier CHARA epochs. These variations are poorly understood and could be caused by changes in size/geometry, mass accretion rate, and gas content in the inner disk. More evidence for MWC 275 variability was recently reported by Wisniewski et al. (2008), who found changes in scattered light intensity between 1998 and 2003-2004.

\subsection{MIR SED and Emission Morphology}

Van Boekel et al. (2005b) analyzed the MIR SED (Fig. 10) of MWC 275 in detail and showed that the SED could be reproduced well with a grain mixture of 1.5 and $0.1 \mu \mathrm{m}$ silicates with mass ratio of $4: 1$. We use results from van Boekel et al. (2005b) in fixing the small grain composition in our disk models.

As seen in Figure 10, the MWC 275 SED falls sharply between 20 and $30 \mu \mathrm{m}$. This drop and the $10 \mu \mathrm{m}$ silicate feature can be simultaneously reproduced only if the mass fraction of the small grain dust component relative to gas beyond $7 \mathrm{AU}$ is less than $20 \%$ of the mass fraction inside of $7 \mathrm{AU}$. If the small grain component is allowed to exist beyond $7 \mathrm{AU}$, then the model far-infrared spectrum becomes much stronger than observed SED. Figure 10 shows a TORUS model SED that fits the MIR and longer wavelength spectrum of MWC 275 well. In this model, $40 \%$ of the $8 \mu \mathrm{m}$ emission arises from the dust rim, with the rim contribution declining to $\sim 20 \%$ at $13 \mu \mathrm{m}$. This model also fits the MIDI VLTI MIR visibilities (Leinert et al. 2004) and reproduces the $0.8 \pm$ 0.1 AU $11 \mu \mathrm{m}$ FWHM minor-axis size of MWC 275 (Fig. 11), naturally explaining why MWC 275 is unresolved by the Keck Segment Tilting Experiment. The quality of the SED and visibility fit in the $8-15 \mu \mathrm{m}$ region is only moderate, probably due to the simple dust composition and distribution that we have assumed in the model. The initial model setup has been chosen to reproduce MWC 275 millimeter interferometry.

Table 8 lists disk parameters for the MWC 275 model and Figure 12 shows the radial distribution of the small grain fractions. The midplane temperature profile and the "flaring" geometry of the disk surface are shown in Figure 13. The dust rim "shadows" (Dullemond et al. 2001; Dullemond \& Dominik 2004) the region of the disk between 0.3 and $1 \mathrm{AU}$, beyond which the disk begins to flare. The $\tau=1$ surface drops down in scale height steeply after 6.5 AU, where the small grain fraction reduces sharply. Our conclusions on dust-grain distribution in the MWC 275 disk are consistent with those of Sitko et al. (2008).

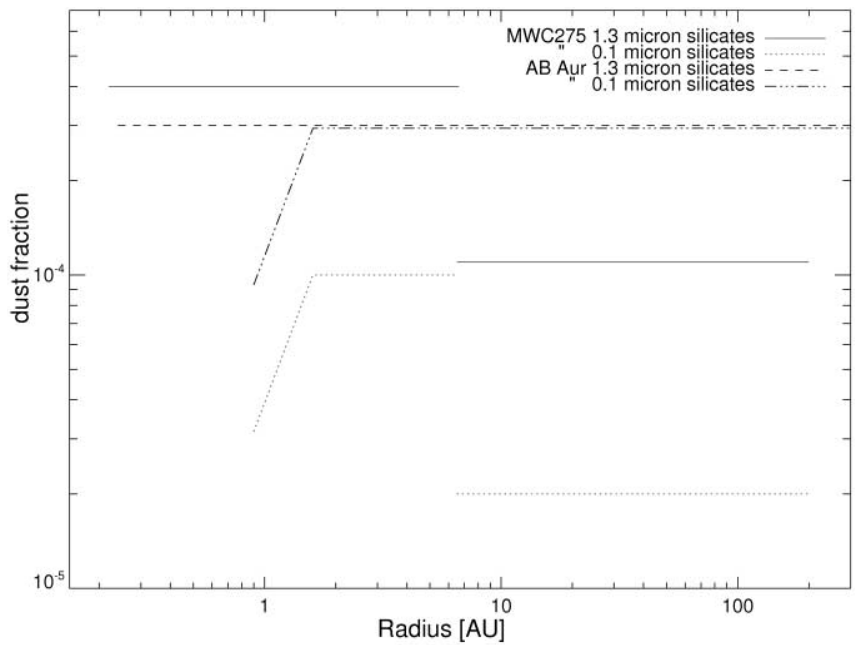

FIG. 12.-Mass fractions of dust components relative to gas. The micron and submicron grain fraction in MWC 275 (gray solid and dotted lines) have to be reduced below $20 \%$ of their values inside of $6.5 \mathrm{AU}$ at larger radii to fit the SED and interferometry. The silicate-grain opacities are from Ossenkopf et al. (1992) and the relative masses of dust grains are from van Boekel et al. (2005b). Between 0.9 and $1.6 \mathrm{AU}, 0.1 \mu \mathrm{m}$ grains are added smoothly to avoid the formation of two distinct dust rims. The bulk of the dust mass is in millimeter-sized grains with a power-law opacity profile (Natta et al. 2004). For AB Aur, we also add a $50 \mu \mathrm{m}$ silicate component to improve SED fits between 40 and $100 \mu \mathrm{m}$. The dust parameters are derived assuming gas and dust are well mixed. [See the electronic edition of the Journal for a color version of this figure.]

\section{AB AUR: ANALYSIS}

AB Aur is a Herbig Ae star (refer to Table 5 for basic properties and photometry) with a total luminosity of $47 L_{\odot}$ (Isella et al. 2006). As in the case of MWC 275, AB Aur's large stellar luminosity dominates the circumstellar disk's energy budget (Garcia Lopez et al. [2006] accretion rates $\leq 10^{-7} M_{\odot} \mathrm{yr}^{-1}$ ). This allows us to ignore accretion heating and model the AB Aur circumstellar disk as a passive disk, reprocessing stellar radiation (Chiang \& Goldreich 1997; Dullemond et al. 2001). For our models, we choose the AB Aur disk mass to be between 0.007 and $0.013 M_{\odot}$ (Lin et al. 2006) and a surface density profile that falls radially as $r^{-1}$ (Corder et al. 2005). The disk outer edge is truncated at 300 AU and the bulk ( $~ 80 \%)$ of the dust mass is assumed to reside in millimeter grains with an opacity that depends on wavelength as $\lambda^{-1}$ for long wavelengths. Here, we describe in detail our modeling results for the NIR and MIR morphology, and the SED of AB Aur.

\subsection{The Thermal NIR Disk}

We follow the procedure outlined in $\S 4.1 .1$ to model the SED and visibilities of $\mathrm{AB}$ Aur. We first attempt to fit a standard curved dust rim model (Fig. 14) to the NIR visibilities. The rim is assumed to be composed of $1.3 \mu \mathrm{m}$ silicate grains and the dust evaporation temperature law is described by equation (3). This produces rim radii too large to fit baselines shorter than $100 \mathrm{~m}$, and we had to increase the $T_{\text {evp }}$ normalization to 2800 from $2000 \mathrm{~K}$. This increases the sublimation temperature at the base of the rim from $\sim 1350$ to $\sim 1950 \mathrm{~K}$. The dashed line in Figure 15 traces the visibility for this model and provides a good fit to the data at baselines shorter than $100 \mathrm{~m}$.

The dust-rim-only model produces large bounces in visibility beyond $150 \mathrm{~m}$ and, as in the case of MWC 275, this bounce is not observed. We have scanned the 150-300 $\mathrm{m}$ baseline (Fig. 15) range several times with CHARA and have failed to detect fringes, ruling out dust-rim-only models for the AB Aur NIR emission. 

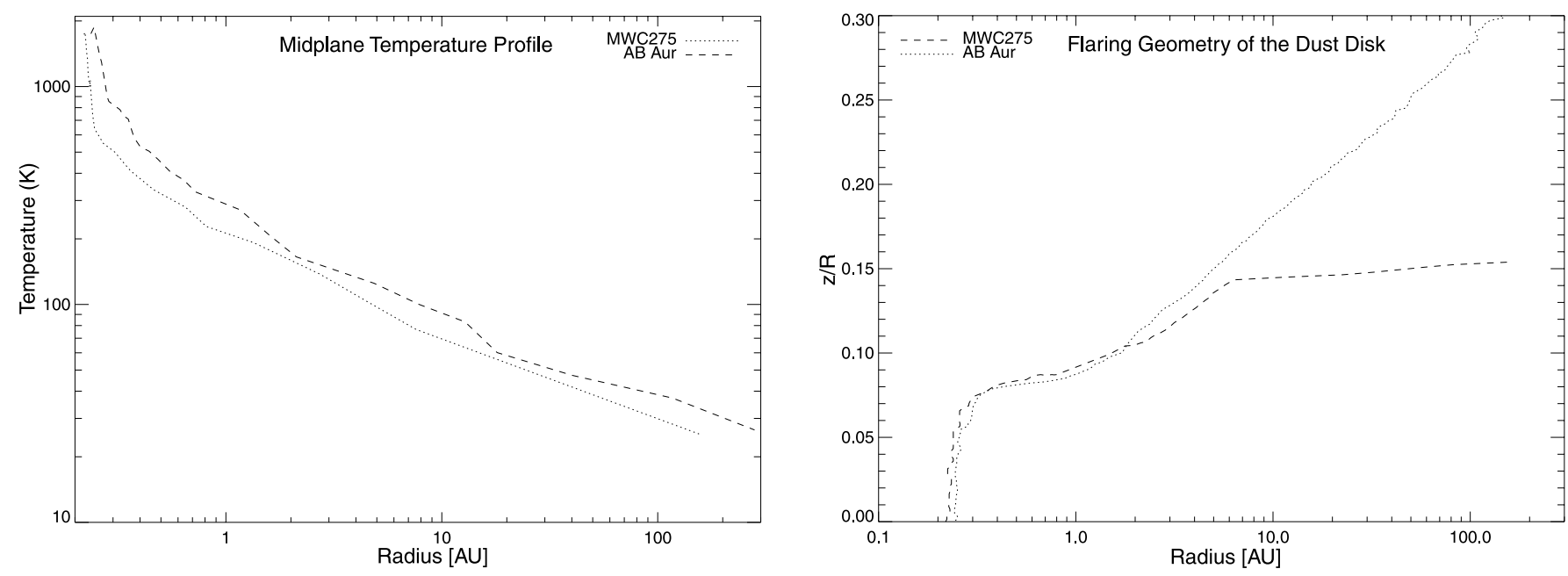

FIG. 13. - Temperature profile and disk-surface shapes for MWC 275 and AB Aur. Left: Midplane temperature profile for MWC 275 (dotted line) and AB Aur (dashed line). Right: $\tau=1$ at $5500 \AA \AA$ surface of the disk measured along radial lines from the central star. The $y$-axis is the polar angle ( 0 is the equatorial plane) in radians.
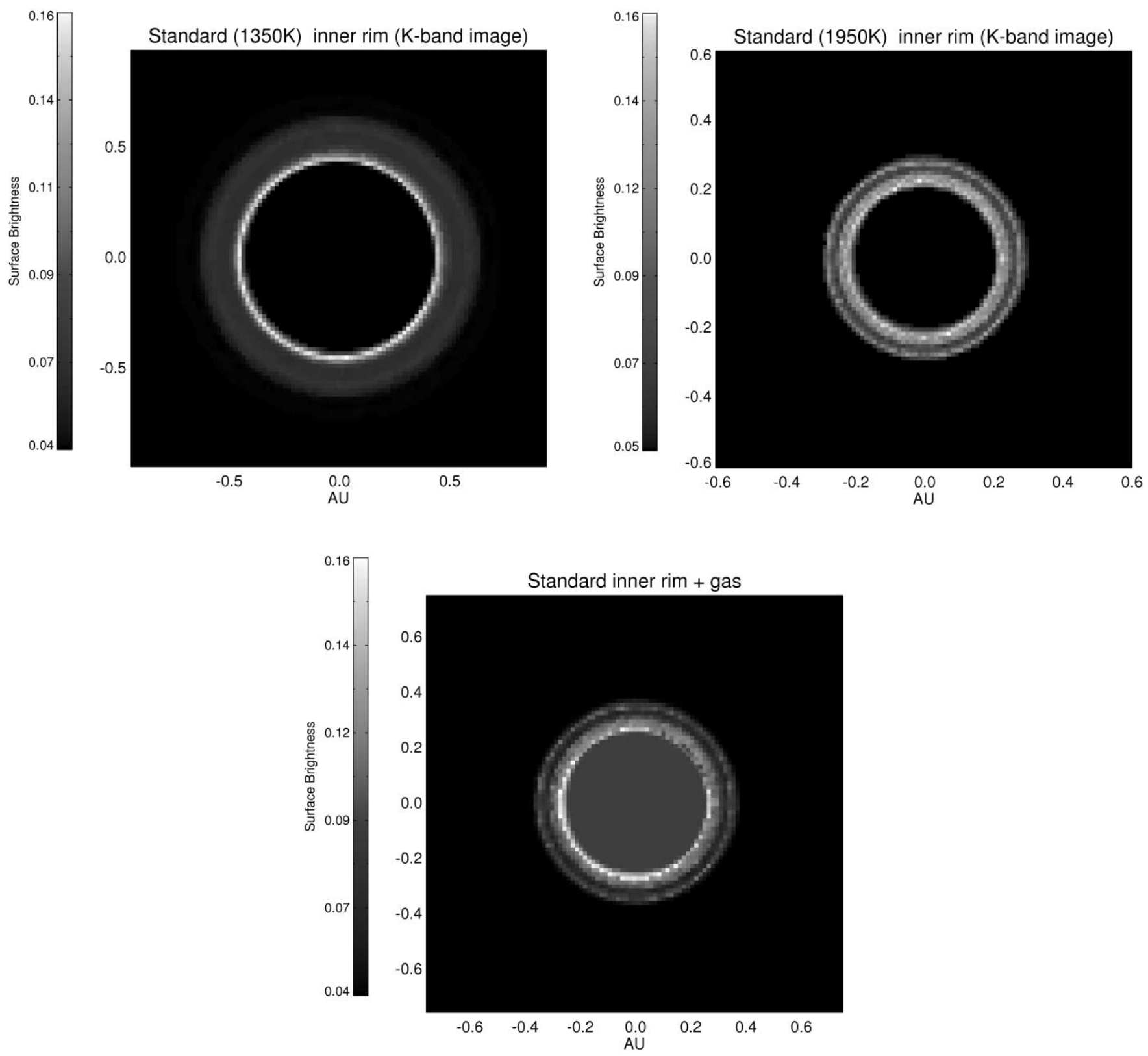

FIG. 14.-Face-on models for NIR emission in AB Aur. Top left: Standard curved dust-rim-only model with rim-base temperature 1350 K. Top right: Standard curved dust-rim-only model with rim-base temperature $\sim 1950 \mathrm{~K}$. Bottom: Curved dust-rim model with gas emission (modeled as a uniform disk centered on the star) added inside the dust rim in to smooth out the emission profile. The central star has been suppressed in all the panels. [See the electronic edition of the Journal for a color version of this figure.] 


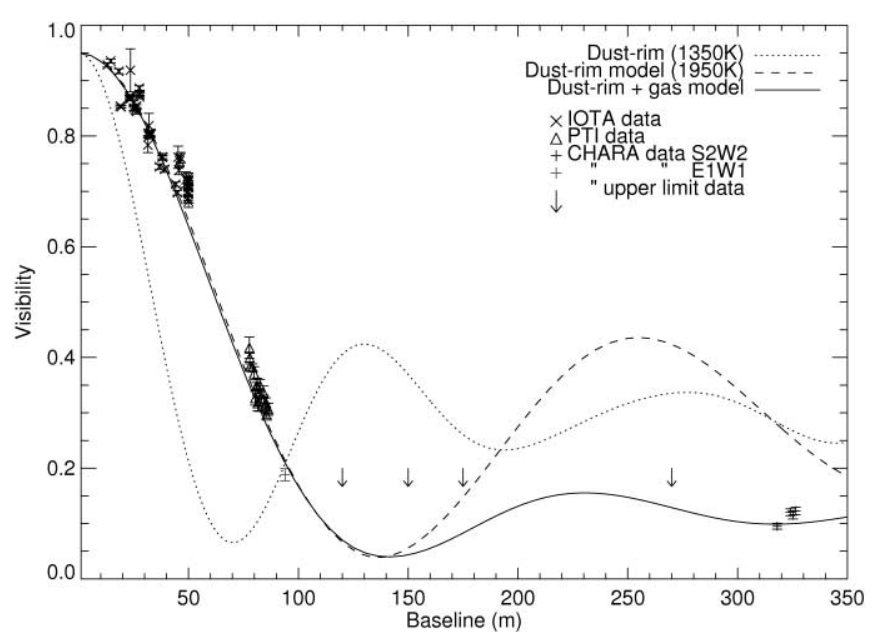

FIG. 15.-AB Aur visibility vs. baseline. The arrows are upper limits on the visibility. The quoted model temperatures are at the base of the dust rims. [See the electronic edition of the Journal for a color version of this figure.]

The addition of a uniform disk of emission interior to the dust destruction radius (Fig. 14, bottom) helps fit the data well (Fig. 15, solid line). The gas component also helps fit the NIR SED (Fig. 16). Parameters for the dust rim + uniform disk model are listed in Table 9 .

\subsection{MIR SED and Emission Morphology}

Liu et al. (2007) resolved the AB Aur disk at $10.3 \mu \mathrm{m}$ using nulling interferometry and measured a disk is inclination of $45^{\circ}-65^{\circ}$ inconsistent with nearly face-on measurements in the millimeter (Corder et al. 2005) and the NIR (Millan-Gabet et al. 2001; Eisner et al. 2004) ranges. Liu et al. (2007) interpreted their result in terms of the AB Aur circumstellar environment being more complicated than a disk. Since AB Aur is well resolved by the Keck Segment Tilting Experiment (Fig. 17), a disk inclination of $45^{\circ}-65^{\circ}$ would have produced observable size difference between the major and minor axes of the disk. We do not find evidence for this size variation in our Segment Tilting data, and hence support a

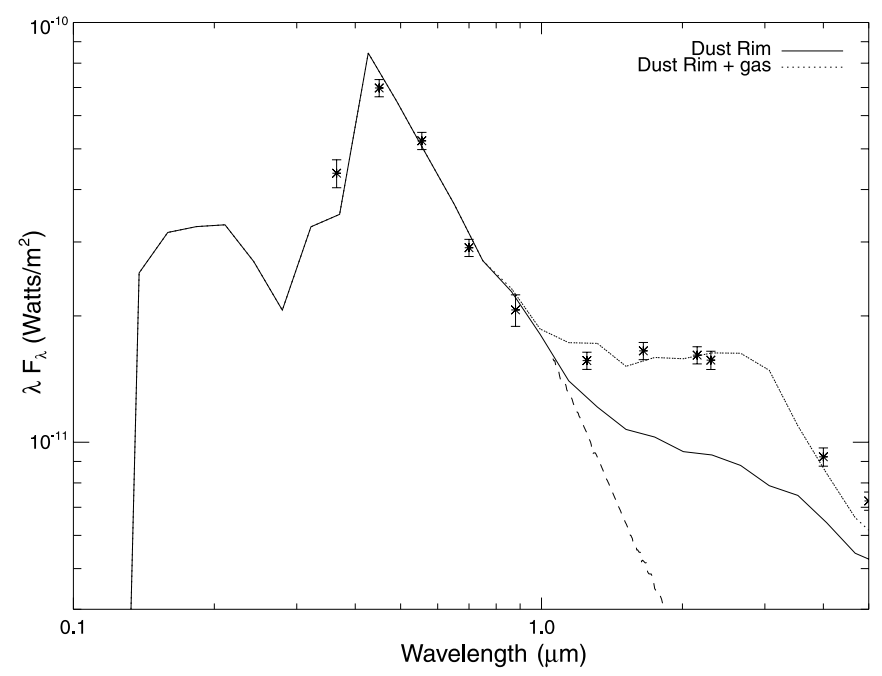

FIG. 16.-NIR SED for AB Aur. The "stars" are photometry points from MDM ( Table 5). The solid line is the SED produced by the star + dust-rim-only model in the top right panel of Fig. 14. The dashed line traces the stellar SED. The dotted line includes emission from gas at $2500 \mathrm{~K}$, assuming that the gas opacity curve derived for MWC 275 (see Fig. 9) is valid for AB Aur as well.
TABLE 9

AB Aur Model-disk Properties Constrained by this Work

\begin{tabular}{|c|c|}
\hline Property & Value \\
\hline \multicolumn{2}{|l|}{ Dust disk } \\
\hline Inner radius & $0.24 \mathrm{AU}^{\mathrm{a}}$ \\
\hline K-band flux contribution from dust rim....... & $22 \%{ }^{\mathrm{a}}$ \\
\hline Mass fractions of dust components ............... & Refer to Fig. 12 \\
\hline
\end{tabular}

NIR gas disk
Surface brightness profile.

Outer radius ......

K-band flux contribution ...

Temperature.

Vertical optical depth.

Gas-opacity profile.

Constant (poorly constrained)
$0.24 \mathrm{AU}^{\mathrm{a}}$
$65 \%$
$>1900 \mathrm{~K}$
$0.14^{\mathrm{a}}$
Refer to Fig. 9

a Tannirkulam et al. (2008). The star contributes $8 \%$ of the K-band flux and an extended envelope (Monnier et al. 2006) contributes 5\%. face-on model for the MIR disk around AB Aur consistent with the NIR and millimeter results.

The MIR spectrum of AB Aur in the 10.7-20 $\mu \mathrm{m}$ range can be modeled well with a dust grain mixture of 1.3 and $0.1 \mu \mathrm{m}$ silicates with equal mass fractions (van Boekel et al. 2005b). In addition to the micron and submicron silicates, we include a $50 \mu \mathrm{m}$ silicate component to model the relatively flat spectrum of AB Aur between 35 and $80 \mu \mathrm{m}$.

Figure 18 shows a TORUS model SED that fits the MIR and longer wavelength spectrum of AB Aur well. In this model, $\sim 40 \%$ of the $8 \mu \mathrm{m}$ emission arises from the dust rim, with the rim contribution declining to $\sim 10 \%$ at $13 \mu \mathrm{m}$. This model also fits the Keck Segment Tilting data visibilities reproducing the $10.5 \pm$ 0.7 AU $10 \mu \mathrm{m}$ FWHM size of AB Aur (Fig. 17). By initial design, the model fits the AB Aur millimeter interferometry and SED.

Table 9 lists disk parameters for the AB Aur model and Figure 12 shows the radial distribution of the small grain fractions. The midplane temperature profile and the $\tau=1$ surface at $5500 \AA$ are shown in Figure 13. The inner rim shadows the disk between 0.3 and $1 \mathrm{AU}$, beyond which the disk surface takes on a flared geometry.

\section{DISCUSSION}

The simultaneous modeling of the infrared and millimeter SED and interferometry of MWC 275 and AB Aur allows us to address several important issues regarding the structure of their circumstellar disks. To maintain clarity in our discussion we divide the disk into two regions: (1) thermal NIR region $(<0.3 \mathrm{AU})$, and (2) outer disk (between 0.3 AU and the disk outer edge).

\subsection{The Thermal NIR Disk}

Detailed modeling ( $\S \S 4.1 .1$ and 5.1) of the inner disk shows that models where the bulk of the NIR emission arises in a dust rim truncated by sublimation fail to fit the long-baseline interferometry data and underestimate the NIR emission by a factor of 2 relative to observations. As mentioned in T08 and demonstrated in detail in this work, the presence of a gas emission component inside the dust destruction radius can solve the interferometry and SED problem simultaneously.

This, however, opens up a number of new questions, namely, (1) what is the geometry of the gas dust transition region? To date there has been no calculation of transition region structure that 

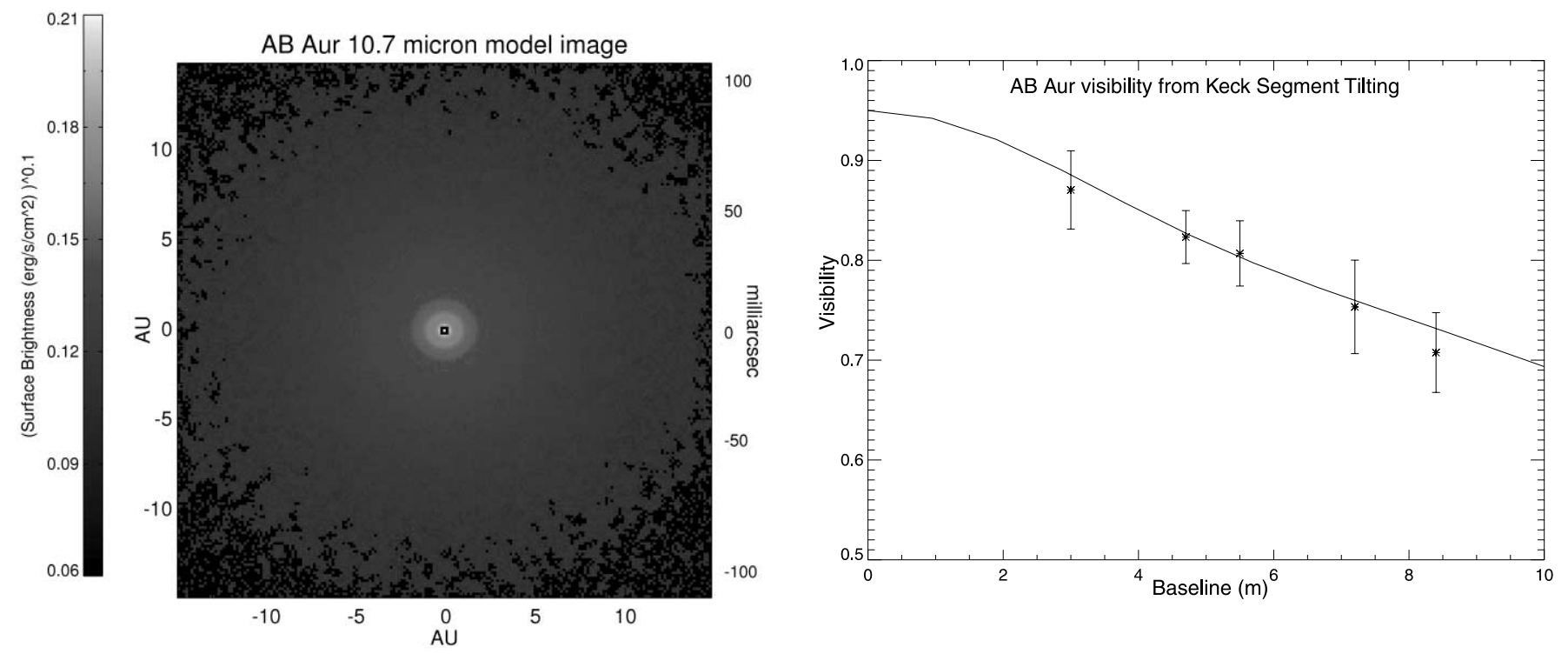

FIG. 17.-A $10.7 \mu \mathrm{m}$ image and visibilities for AB Aur. Left: Synthetic $10.7 \mu \mathrm{m}$ TORUS image. Right: Model visibilities (solid line) compared with azimuthally averaged Keck Segment Tilting data (Monnier et al. 2004). The model also includes 5\% emission arising from an extended envelope. [See the electronic edition of the Journal for a color version of this figure.]

treats both gas and dust simultaneously in a self consistent manner. (2) What are the relative contributions of accretion and stellar radiation to heating the gas? We have shown that an ad hoc addition of an NIR emission component inside the dust destruction radius helps explain the data, but the current modeling does not shed any light on the energy budget question. (3) What are the gas species that provide the NIR opacity? Is the opacity molecular in nature or is it from free-free and free-bound processes? If a significant portion of the NIR emission were indeed arising from molecular gas, then Figure 9 shows that theoretical gas opacities depend much more sensitively on wavelength between 4 and $10 \mu \mathrm{m}$ than what is observed. This suggests that some of the molecules providing the model opacities might be getting destroyed by the stellar UV radiation field.

In the course of modeling the MWC 275 and AB Aur disks, we realized ( $\S 4.1 .1)$ that the observed $K$-band sizes could be reproduced only if the dust sublimation temperature at the base of

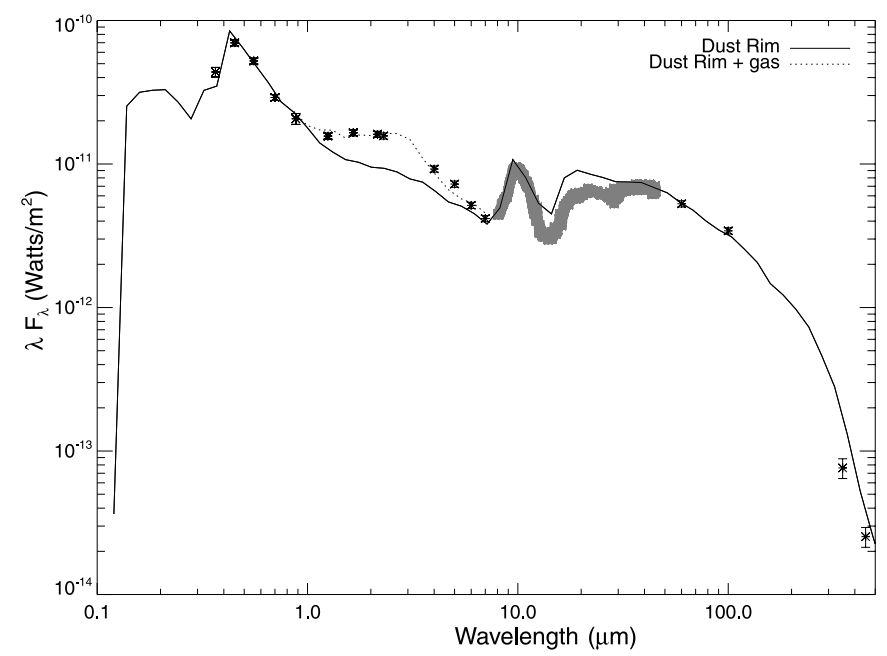

FIG. 18.- AB Aur SED from UV to millimeter. The MIR, far-infrared, and submillimeter data are from Meeus et al. (2001 and references therein). The solid line traces the dust-disk model SED (see $\S 5.2$ ). The dotted line traces the dust-disk + gas model. The relative contributions of star, dust, and gas to the total integrated flux are $0.67,0.27$, and 0.06 , respectively. the dust rims were increased to $\sim 1850 \mathrm{~K}$ from the experimentally measured silicate evaporation temperatures of $\sim 1400 \mathrm{~K}$ (Pollack et al. 1994). A simple treatment of the gas-dust transition region by Muzerolle et al. (2004) suggests that gas is not effective in modifying rim geometry. In the absence of shielding by gas, the large dust sublimation temperatures indicate that the grains in the inner disks of young stars are significantly more refractory and/or optically transparent than has been assumed in the literature. There is also the possibility that the gas gets optically thick along the midplane, shielding the dust from direct stellar radiation and allowing the dust rim to exist closer to the star (Monnier et al. 2005; Isella et al. 2006). Future, high-resolution NIR spectroscopic studies of MWC 275 and AB Aur, combined with selfconsistent models of the gas density and temperature structure, will help address many of the questions raised here.

MWC 275 and $\mathrm{AB}$ Aur require gas emission to explain the their SED interferometry. In contrast, past modeling work by Isella et al. (2006) seems to suggest that dust rims alone are probably sufficient to explain the NIR data on the young stars V1295 Aq1 (A2 IVe) and CQ Tau (F2 IVe). A larger sample of young stars will therefore have to be observed with milliarcsecond interferometry to establish and understand trends between spectral type, stellar mass, accretion rates, and the contribution of gas emission to NIR SED.

A new and exciting observational domain will be opened with the commissioning of the fringe tracker (Berger et al. 2006) for CHARA MIRC (Monnier et al. 2007) in the summer of 2008. This will sufficiently improve CHARA MIRC sensitivities to combine light from three or more telescopes, allowing the first milliarcsecond non-parametric imaging of MWC 275 and AB Aur in the NIR. The snapshot multiple-baseline coverage will provide us a powerful tool in understanding the infrared time variability of YSO disks.

\subsection{The Outer Disk}

Our models for the MWC 275 and AB Aur MIR interferometry and SED suggest that the outer disks of these systems are at different evolutionary stages. MWC $27510 \mu \mathrm{m}$ size and MIR SED can only be reproduced if the disk is depleted in micron and submicron sized grains beyond $\sim 7 \mathrm{AU}(\S 4.2)$. This meshes well 
with the fact that the observed $10.7 \mu \mathrm{m}$ size of MWC 275 is $\sim 3$ times smaller than AB Aur. The depletion of small grains beyond $7 \mathrm{AU}$ in the disk atmosphere indicates that the dust particles in MWC 275 have undergone significant settling. However, the presence of the $10 \mu \mathrm{m}$ silicate feature in MWC $275 \mathrm{im}-$ plies that there is some process (like planetesimal collisions) that maintains the supply of micron-sized grains in the inner regions of the disk.

Our models predict that the inner dust rim shadows (Dullemond $\&$ Dominik 2004) the region of the disk between 0.3 and 1 AU. The structure and size of the shadow depends sensitively on the composition of grains in the circumstellar disk (Tannirkulam et al. 2007) and hence is an important probe of dust physics. The presence of the shadow has not been observationally confirmed yet in any YSO system, although some indirect evidence has been found in VV Ser (Pontoppidan et al. 2007).

\section{CONCLUSIONS}

We have presented the first set of comprehensive disk models for the SED and interferometry of Herbig Ae stars MWC 275 and AB Aur. We have shown that "standard" models for the dust evaporation front where the bulk of the NIR emission arises from a dust wall fail to explain the NIR SED and interferometry. Standard models produce large bounces in visibility at high spatial frequency, which is not observed in the data. We have conclusively demonstrated that the presence of an additional smooth emission component (presumably hot gas) inside the dust destruction ra- dius and on a similar size scale to the dust rim can ameliorate the situation. In the absence of shielding of starlight by gas, we have established that dust grains in the gas-dust transition region will have to be highly refractory, sublimating at $1850 \mathrm{~K}$. The small MIR size of MWC 275 relative to AB Aur shows that the dust grains in the outer disk MWC 275 are significantly more evolved/ settled than the grains in the AB Aur disk. We suggest that dynamical processes (such as planetesimal collisions) that maintain the population of micron-sized grains producing the $10 \mu \mathrm{m}$ feature in the spectrum are operational only in the inner $7 \mathrm{AU}$ of MWC 275. However, in AB Aur the small-dust producing mechanisms exist at least out to $20 \mathrm{AU}$ and maybe even beyond.

A. T. acknowledges contributions from Nuria Calvet, Michael Busha, Marlin Whitaker, and Steve Golden. Research at the CHARA array is supported by the National Science Foundation through grants AST 06-06958 and AST 03-52723 and by the Georgia State University through the offices of the Dean of the College of Arts and Sciences and the Vice President for Research. This project was partially supported by NASA grant 050283 . This publication makes use of NASA's Astrophysics Data System Abstract Service. CHARA visibility-calibrator sizes were obtained with the $\mathrm{fBol}$ module of getCal, a package made available by the Michelson Science Center, California Institute of Technology (http://msc.caltech.edu). Computations were performed on the Legato-Opus Cluster Network at the University of Michigan.

\section{APPENDIX A}

\section{A1. PHOTOMETRY}

Optical and near-infrared photometry for a number of YSOs, and a few main-sequence and evolved stars are presented in Tables 10 and 11. The data were collected at the MDM Observatory between 2004 November and 2006 August.

TABLE 10

UBVRI РнOTOMETRY

\begin{tabular}{|c|c|c|c|c|c|c|c|c|}
\hline Target & $\begin{array}{l}\text { R.A. } \\
\text { (J2000.0) }\end{array}$ & Decl. & $U$ & $B$ & V & $R$ & $I$ & $\begin{array}{c}\text { UT Date } \\
\text { of Observation }\end{array}$ \\
\hline HIP $2080 \ldots \ldots . . .$. & 002616.5 & +034933 & $\cdots$ & $6.72 \pm 0.05$ & $6.81 \pm 0.04$ & $6.94 \pm 0.06$ & $6.93 \pm 0.04$ & 2006 Aug 27 \\
\hline 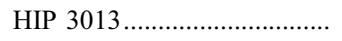 & 003820.3 & -145954 & $\ldots$ & $10.67 \pm 0.05$ & $10.86 \pm 0.04$ & $6.95 \pm 0.06$ & $11.07 \pm 0.04$ & 2006 Aug 27 \\
\hline 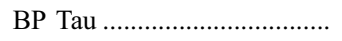 & 041915.8 & +290627 & $13.20 \pm 0.15$ & $13.36 \pm 0.03$ & $12.32 \pm 0.04$ & $11.45 \pm 0.04$ & $10.60 \pm 0.04$ & 2004 Nov 29 \\
\hline 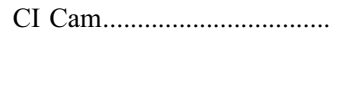 & 041942.1 & +555957 & $\begin{array}{l}12.13 \pm 0.07 \\
12.32 \pm 0.08\end{array}$ & $\begin{array}{l}12.41 \pm 0.06 \\
12.70 \pm 0.04 \\
12.59 \pm 0.05\end{array}$ & $\begin{array}{l}11.77 \pm 0.05 \\
11.75 \pm 0.05 \\
11.71 \pm 0.03\end{array}$ & $\begin{array}{l}10.79 \pm 0.05 \\
10.64 \pm 0.05 \\
10.72 \pm 0.04\end{array}$ & $\begin{array}{l}9.99 \pm 0.04 \\
9.82 \pm 0.09 \\
9.94 \pm 0.05\end{array}$ & $\begin{array}{l}2004 \text { Nov } 27 \\
2005 \text { Dec } 10 \\
2006 \text { Aug } 28\end{array}$ \\
\hline DG Tau & 042704.7 & +260616 & $13.93 \pm 0.04$ & $13.97 \pm 0.03$ & $12.79 \pm 0.04$ & $11.70 \pm 0.04$ & $10.67 \pm 0.04$ & 2004 Nov 29 \\
\hline DI Tau. & 042942.5 & +263249 & $16.06 \pm 0.25$ & $14.45 \pm 0.03$ & $12.96 \pm 0.04$ & $11.87 \pm 0.04$ & $10.70 \pm 0.04$ & 2004 Nov 29 \\
\hline V830 Tau. & 043310.0 & +243343 & $14.66 \pm 0.15$ & $13.52 \pm 0.03$ & $12.21 \pm 0.04$ & $11.26 \pm 0.04$ & $10.44 \pm 0.04$ & 2004 Nov 29 \\
\hline LkCa 15 & 043917.8 & +222103 & $13.98 \pm 0.07$ & $13.30 \pm($ & $12.09 \pm$ & $11.26 \pm$ & $10.52 \pm$ & 2004 Nov 29 \\
\hline 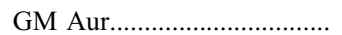 & 045511.0 & +302159 & $13.90 \pm 0.04$ & $13.38 \pm($ & $12.19 \pm$ & $11.34 \pm 0.04$ & $10.61 \pm$ & 2004 Nov 29 \\
\hline AB Aur. & 045545.8 & +30 & $\begin{array}{c}7.18 \pm 0.08 \\
\ldots\end{array}$ & $\begin{array}{l}7.14 \pm \\
7.19 \pm\end{array}$ & $\begin{array}{l}7.01= \\
7.05=\end{array}$ & $\begin{array}{l}6.96 \pm \\
7.01 \pm\end{array}$ & $\begin{array}{l}6.70= \\
6.80=\end{array}$ & $\begin{array}{l}2005 \text { Dec } 10 \\
2006 \text { Aug } 27\end{array}$ \\
\hline GW Ori .. & 052908.4 & +115213 & $11.60 \pm 0.04$ & $11.29 \pm 0.03$ & $10.16 \pm 0.04$ & $9.38 \pm 0.04$ & $8.75 \pm 0.04$ & 2004 Nov 29 \\
\hline 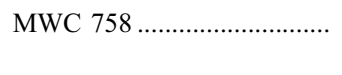 & 053027.5 & +251957 & $8.59 \pm 0.07$ & $\begin{array}{l}8.56 \pm 0.06 \\
8.58 \pm 0.05\end{array}$ & $\begin{array}{l}8.28 \pm 0.05 \\
8.28 \pm 0.03\end{array}$ & $\begin{array}{l}8.11 \pm 0.05 \\
8.15 \pm 0.04\end{array}$ & $\begin{array}{l}7.95 \pm 0.04 \\
7.92 \pm 0.05\end{array}$ & $\begin{array}{l}2004 \text { Nov } 29 \\
2006 \text { Aug } 28\end{array}$ \\
\hline V380 Ori* & 053625.4 & -064258 & $\begin{array}{l}10.29 \pm 0.07 \\
10.07 \pm 0.08\end{array}$ & $\begin{array}{l}10.43 \pm 0.06 \\
10.15 \pm 0.04\end{array}$ & $\begin{aligned} 10.06 & \pm 0.05 \\
9.74 & \pm 0.04\end{aligned}$ & $\begin{array}{l}9.67 \pm 0.05 \\
9.38 \pm 0.05\end{array}$ & $\begin{array}{l}8.99 \pm 0.04 \\
8.80 \pm 0.09\end{array}$ & $\begin{array}{l}2004 \text { Nov } 29 \\
2008 \text { Aug } 27\end{array}$ \\
\hline
\end{tabular}


TABLE 10-Continued

\begin{tabular}{|c|c|c|c|c|c|c|c|c|}
\hline \multirow[t]{2}{*}{ 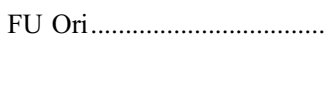 } & 054522.4 & +090412 & & $10.80 \pm 0.08$ & $9.52 \pm 0.15$ & $8.63 \pm 0.05$ & $8.11 \pm 0.15$ & 2004 Nov 29 \\
\hline & & & & $10.87 \pm 0.05$ & $9.65 \pm 0.03$ & $8.87 \pm 0.05$ & $8.13 \pm 0.05$ & 2006 Aug 28 \\
\hline \multirow[t]{2}{*}{ HD $45677 \ldots \ldots \ldots$} & 062817.4 & -130311 & $7.03 \pm 0.07$ & $7.56 \pm 0.06$ & $7.51 \pm 0.05$ & $7.33 \pm 0.05$ & $7.24 \pm 0.04$ & 2004 Nov 27 \\
\hline & & & $6.99 \pm 0.08$ & & $7.52 \pm 0.04$ & $7.35 \pm 0.05$ & $7.06 \pm 0.09$ & 2005 Dec 10 \\
\hline MWC 166. & 070425.5 & -102716 & $7.08 \pm 0.07$ & $7.55 \pm 0.06$ & $7.16 \pm 0.05$ & $6.97 \pm 0.05$ & $6.65 \pm 0.04$ & 2004 Nov 27 \\
\hline HD 58647 . & 072556.1 & $-14 \quad 1044$ & $6.66 \pm 0.07$ & $6.874 \pm 0.06$ & $6.73 \pm 0.05$ & $6.76 \pm 0.05$ & $6.67 \pm 0.04$ & 2004 Nov 27 \\
\hline IRC $+10216 .$. & 094759.4 & +131644 & $\ldots$ & $\ldots$ & $>15.9$ & $15.60 \pm 0.10$ & $12.52 \pm 0.04$ & 2004 Nov 27 \\
\hline Beta Leo................................. & 114903.6 & +143419 & $\ldots$ & $2.36 \pm 0.09$ & $2.18 \pm 0.08$ & $2.17 \pm 0.09$ & $2.22 \pm 0.07$ & 2006 Jun 9 \\
\hline \multirow[t]{2}{*}{ HD 141569} & 154957.8 & -035516 & $\ldots$ & $7.32 \pm 0.09$ & $7.16 \pm 0.08$ & $7.14 \pm 0.09$ & $7.15 \pm 0.07$ & 2006 Jun 9 \\
\hline & & & $\ldots$ & $7.32 \pm 0.05$ & $7.14 \pm 0.04$ & $7.23 \pm 0.06$ & $7.03 \pm 0.04$ & 2006 Aug 27 \\
\hline HD $142666 .$. & 155640.0 & -220140 & $\ldots$ & $9.70 \pm 0.05$ & $9.05 \pm 0.04$ & $8.77 \pm 0.06$ & $8.32 \pm 0.04$ & 2006 Aug 28 \\
\hline \multirow[t]{2}{*}{ HD 150193 (MWC 863)..... } & 164017.9 & -235345 & $\ldots$ & $9.43 \pm 0.09$ & $8.87 \pm 0.08$ & $8.48 \pm 0.09$ & $8.20 \pm 0.07$ & 2006 Jun 9 \\
\hline & & & $\ldots$ & $9.42 \pm 0.05$ & $8.87 \pm 0.04$ & $8.58 \pm 0.06$ & $8.04 \pm 0.04$ & 2006 Aug 27 \\
\hline KKOph.. & 171008.0 & -271518 & $\ldots$ & $12.77 \pm 0.09$ & $12.11 \pm 0.08$ & $11.61 \pm 0.09$ & $11.16 \pm 0.07$ & 2006 Jun 9 \\
\hline 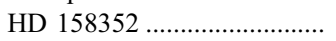 & 172849.7 & +001950 & $\ldots$ & $5.67 \pm 0.05$ & $5.38 \pm 0.04$ & $5.33 \pm 0.06$ & $5.18 \pm 0.04$ & 2006 Aug 27 \\
\hline \multirow[t]{2}{*}{ 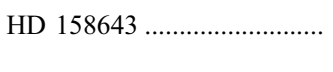 } & 173125.0 & -235745 & $\ldots$ & $4.81 \pm 0.09$ & $4.78 \pm 0.08$ & $4.80 \pm 0.09$ & $4.73 \pm 0.07$ & 2006 Jun 9 \\
\hline & & & & $4.87 \pm 0.05$ & $4.78 \pm 0.03$ & $4.81 \pm 0.04$ & $4.62 \pm 0.04$ & 2006 Aug 28 \\
\hline RSOph.... & 175013.2 & -064228 & $\ldots$ & $12.65 \pm 0.08$ & $11.43 \pm 0.06$ & $10.21 \pm 0.05$ & $9.51 \pm 0.07$ & 2006 Jun 8 \\
\hline \multirow{2}{*}{ MWC $275 \ldots \ldots \ldots$} & 175621.3 & -215722 & & $6.98 \pm 0.08$ & $6.84 \pm 0.06$ & $6.86 \pm 0.05$ & $6.71 \pm 0.07$ & 2006 Jun 8 \\
\hline & & & & $7.01 \pm 0.05$ & $6.86 \pm 0.04$ & $6.90 \pm 0.06$ & $6.72 \pm 0.04$ & 2006 Aug 27 \\
\hline HD $169412 .$. & 182133.5 & +525408 & & $7.89 \pm 0.09$ & $7.82 \pm 0.08$ & $7.85 \pm 0.09$ & $7.97 \pm 0.07$ & 2006 Jun 9 \\
\hline \multirow[t]{2}{*}{ MWC $297 \ldots \ldots \ldots$} & 182739.6 & -034952 & & $14.38 \pm 0.09$ & $12.26 \pm 0.08$ & $10.29 \pm 0.09$ & $9.10 \pm 0.07$ & 2006 Jun 9 \\
\hline & & & & $14.27 \pm 0.05$ & $12.23 \pm 0.04$ & $10.30 \pm 0.06$ & $8.92 \pm 0.04$ & 2006 Aug 27 \\
\hline VVSer... & 182847.9 & +000840 & & $13.23 \pm 0.08$ & $12.22 \pm 0.06$ & $11.36 \pm 0.05$ & $10.61 \pm 0.07$ & 2006 Jun 8 \\
\hline MWC 342. & 202303.6 & +392950 & $\ldots$ & $11.88 \pm 0.09$ & $10.57 \pm 0.08$ & $9.44 \pm 0.09$ & $8.72 \pm 0.07$ & 2006 Jun 9 \\
\hline V1057 Cyg................ & 205853.7 & +441528 & $16.00 \pm 0.28$ & $14.32 \pm 0.06$ & $12.50 \pm 0.05$ & $11.18 \pm 0.05$ & $9.78 \pm 0.04$ & 2004 Nov 27 \\
\hline MWC 361 & 210136.9 & +680948 & $\ldots$ & $7.75 \pm 0.05$ & $7.33 \pm 0.03$ & $7.00 \pm 0.04$ & $6.62 \pm 0.05$ & 2006 Aug 28 \\
\hline AS $477 \ldots \ldots \ldots$ & 215233.9 & +471338 & $10.48 \pm 0.07$ & $10.52 \pm 0.06$ & $10.07 \pm 0.05$ & $9.85 \pm 0.05$ & $9.50 \pm 0.04$ & 2004 Nov 27 \\
\hline 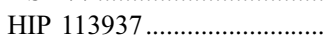 & 230423.6 & -240656 & $\ldots$ & $9.12 \pm 0.05$ & $9.07 \pm 0.04$ & $9.18 \pm 0.06$ & $9.08 \pm 0.04$ & 2006 Aug 27 \\
\hline 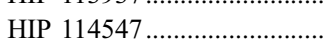 & $23 \quad 1209.4$ & -252414 & $\cdots$ & $9.51 \pm 0.05$ & $9.12 \pm 0.04$ & $8.98 \pm 0.06$ & $8.67 \pm 0.04$ & 2006 Aug 27 \\
\hline 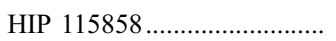 & 232825.2 & $-25 \quad 2514$ & $\ldots$ & $7.09 \pm 0.05$ & $6.88 \pm 0.04$ & $6.92 \pm 0.06$ & $6.72 \pm 0.04$ & 2006 Aug 27 \\
\hline
\end{tabular}

Notes.- Majority of the MDM targets are YSOs. Units of right ascension are hours, minutes, and seconds, and units of declination are degrees, arcminutes, and arcseconds. 
TABLE 11

$J H K$ РHOTOMETRY

\begin{tabular}{|c|c|c|c|c|c|c|}
\hline Target & $\begin{array}{c}\text { R.A. } \\
\text { (J2000.0) }\end{array}$ & Decl. & $J$ & $H$ & $K$ & UT Date of Observation \\
\hline V892 Tau ... & 041840.6 & +281916 & $8.61 \pm 0.05$ & $7.08 \pm 0.05$ & $5.86 \pm 0.05$ & 2005 Dec 17 \\
\hline & & & $9.05 \pm 0.05$ & $8.32 \pm 0.05$ & $7.85 \pm 0.05$ & 2005 Dec 17 \\
\hline \multirow[t]{2}{*}{ CI Cam } & 041942.1 & +555958 & $7.20 \pm 0.10$ & $5.68 \pm 0.10$ & $4.44 \pm 0.10$ & 2004 Dec 1 \\
\hline & & & $7.01 \pm 0.05$ & $5.63 \pm 0.05$ & $4.35 \pm 0.05$ & 2005 Dec 17 \\
\hline LkCa 15 & 043917.8 & +222104 & $9.33 \pm 0.05$ & $8.72 \pm 0.05$ & $8.23 \pm 0.05$ & 2005 Dec 17 \\
\hline 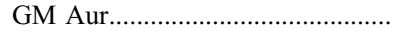 & 045511.0 & +302200 & $9.40 \pm 0.05$ & $8.80 \pm 0.05$ & $8.52 \pm 0.05$ & 2005 Dec 17 \\
\hline 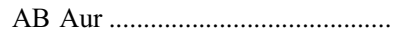 & 045545.8 & +303304 & $5.99 \pm 0.05$ & $5.28 \pm 0.05$ & $4.37 \pm 0.05$ & 2005 Dec 17 \\
\hline MWC 480 & 045846.3 & +295037.0 & $6.90 \pm 0.05$ & $6.38 \pm 0.05$ & $5.57 \pm 0.05$ & 2005 Dec 17 \\
\hline \multirow[t]{2}{*}{ RW Aur } & 050749.5 & +302405 & $8.22 \pm 0.05$ & $7.67 \pm 0.05$ & $7.16 \pm 0.05$ & 2004 Dec 1 \\
\hline & & & $8.34 \pm 0.10$ & $7.66 \pm 0.10$ & $7.18 \pm 0.10$ & 2005 Dec 17 \\
\hline GW Ori $\ldots$ & 052908.4 & +115213 & $7.42 \pm 0.05$ & $6.67 \pm 0.05$ & $5.83 \pm 0.05$ & 2005 Dec 17 \\
\hline 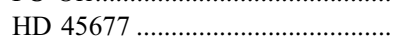 & 062817.4 & -130311 & $6.85 \pm 0.05$ & $6.22 \pm 0.05$ & $4.61 \pm 0.05$ & 2005 Dec 17 \\
\hline MWC $147 \ldots \ldots \ldots \ldots$ & 063305.2 & +101920 & $7.34 \pm 0.05$ & $6.70 \pm 0.05$ & $5.73 \pm 0.05$ & 2005 Dec 17 \\
\hline Z CMa & 070343.2 & -113306 & $6.66 \pm 0.05$ & $5.45 \pm 0.05$ & $3.94 \pm 0.05$ & 2005 Dec 17 \\
\hline MWC 166 & 070425.5 & -102716 & $6.32 \pm 0.05$ & $6.32 \pm 0.05$ & $6.27 \pm 0.05$ & 2005 Dec 17 \\
\hline 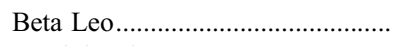 & 114903.6 & +143419 & $1.92 \pm 0.08$ & $1.96 \pm 0.07$ & $1.90 \pm 0.08$ & 2006 Jun 2 \\
\hline 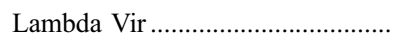 & 141906.6 & -132216 & $4.26 \pm 0.08$ & $4.25 \pm 0.07$ & $4.20 \pm 0.08$ & 2006 Jun 2 \\
\hline HD 141569 & 154957.8 & -035516 & $6.67 \pm 0.08$ & $6.54 \pm 0.07$ & $6.48 \pm 0.08$ & 2006 Jun 2 \\
\hline HD 143006 & 155836.9 & -225715 & $8.18 \pm 0.08$ & $7.57 \pm 0.07$ & $5.94 \pm 0.08$ & 2006 Jun 2 \\
\hline HD 144432 & 160658.0 & -274310 & $7.23 \pm 0.08$ & $6.69 \pm 0.07$ & $6.14 \pm 0.08$ & 2006 Jun 2 \\
\hline HD 150193 (MWC 863)............... & 164017.9 & -235345 & $6.84 \pm 0.08$ & $6.02 \pm 0.07$ & $5.13 \pm 0.08$ & 2006 Jun 2 \\
\hline 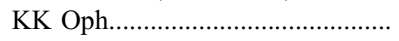 & 171008.1 & -271518 & $8.46 \pm 0.08$ & $7.09 \pm 0.07$ & $5.71 \pm 0.08$ & 2006 Jun 2 \\
\hline 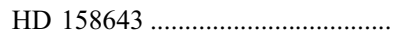 & 173125.0 & -235746 & $4.76 \pm 0.08$ & $4.63 \pm 0.07$ & $4.34 \pm 0.08$ & 2006 Jun 2 \\
\hline 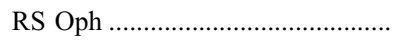 & 175013.2 & -064229 & $7.94 \pm 0.08$ & $7.17 \pm 0.07$ & $6.77 \pm 0.08$ & 2006 Jun 4 \\
\hline MWC 342 & 202303.6 & +392950 & $7.01 \pm 0.05$ & $5.98 \pm 0.05$ & $4.78 \pm 0.05$ & 2005 Dec 17 \\
\hline & & & $6.94 \pm 0.08$ & $5.92 \pm 0.08$ & $4.65 \pm 0.08$ & 2006 Jun 3 \\
\hline 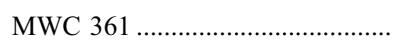 & 210136.9 & +680948 & $6.12 \pm 0.05$ & $5.58 \pm 0.05$ & $4.77 \pm 0.05$ & 2005 Dec 17 \\
\hline 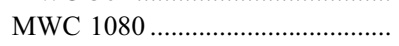 & 231725.6 & +605043 & $7.38 \pm 0.05$ & $6.04 \pm 0.05$ & $4.68 \pm 0.05$ & 2005 Dec 17 \\
\hline
\end{tabular}

Notes.-Majority of the MDM targets are YSOs. Units of right ascension are hours, minutes, and seconds, and units of declination are degrees, arcminutes, and arcseconds.

\section{A2. "EFFECTIVE BASELINES" AS A TOOL IN CHARACTERIZING VISIBILITY INFORMATION ON MWC 275}

Let $B_{\text {projected }}$ be the projected interferometric baseline and let $V\left(B_{\text {projected }}\right)$ be the visibility for a circularly symmetric brightness distribution. For a flat disk inclined at angle $\phi$ and oriented at some P.A., we plotted $V\left(B_{\text {eff }}\right)$ in Figure 5 . The effective baseline $B_{\text {eff }}$ is defined as

$$
B_{\text {eff }}=B_{\text {projected }} \sqrt{\cos ^{2}(\theta)+\cos ^{2}(\phi) \sin ^{2}(\theta)}
$$

where $\theta$ is the angle between the $u v$ vector for the observation and the major axis of the inclined disk and $\phi$ is the inclination of the disk $\left(0^{\circ}\right.$ inclination is face-on). Effective baselines account for the decrease in interferometric resolution due to the inclination of the disk in the sky. They capture the geometry of flat disks correctly, but the geometry of finitely thick disks is represented only approximately (optical depth effects and 3D geometry of thick disks are not taken into account). Here, we argue that effective baselines are good (albeit approximate) tools for capturing details of the MWC 275 disk geometry.

In order to determine the inclination angle and sky orientation of the disk, we adopted the following procedure. MWC 275 visibility values measured with W1W2, S2W2, and E2S2 CHARA telescope pairs are close to and just prior to the first minimum in the visibility 


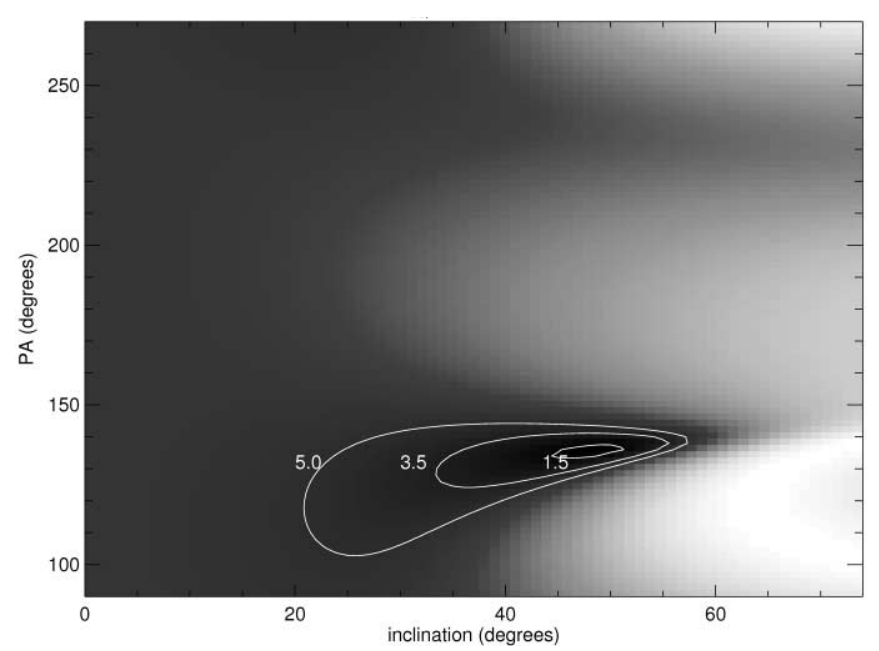

FIG. 19.- - Reduced $\chi^{2}$ surface for the linear fits to the observed visibilities (obtained with the W1W2, S2W2, and E2S2 CHARA telescope pairs) as a function of effective interferometric baseline. The solid curves are reduced $\chi^{2}$ contours of $5,3.5$, and 1.5 , respectively. [See the electronic edition of the Journal for a color version of this figure.]

curve (see Fig. 5). In this region the visibility-baseline relation for the emission models in Figure 6 can be approximated with a linear function. We calculated reduced $\chi^{2}$ values for the best-fit line to the W1W2, S2W2, and E2S2 visibilities as a function of effective baseline, varying the assumed inclination and P.A. of the observed disk. Figure 19 shows the reduced $\chi^{2}$ surface for the fits, plotted against the assumed disk inclination and P.A.. To further illustrate the change in quality of fits as inclination and P.A.s are varied, Figure 20 shows the linear fits to the visibility versus effective baseline data set.

As seen in Figures 19 and 20, the quality of the fits show dramatic improvement at MWC 275 disk P.A. of $136^{\circ} \pm 2^{\circ}$ and inclination of $48^{\circ} \pm 2^{\circ}$. These values are very close to a disk P.A. of $139^{\circ} \pm 15^{\circ}$ and inclination of $51_{-9}^{+11}$ degrees determined in Wassell et al. (2006). The excellent agreement in inclination and P.A. values for MWC 275 from two independent methods strongly supports a disk model for MWC 275, validating the use of "effective baselines" to plot MWC 275 visibilities.
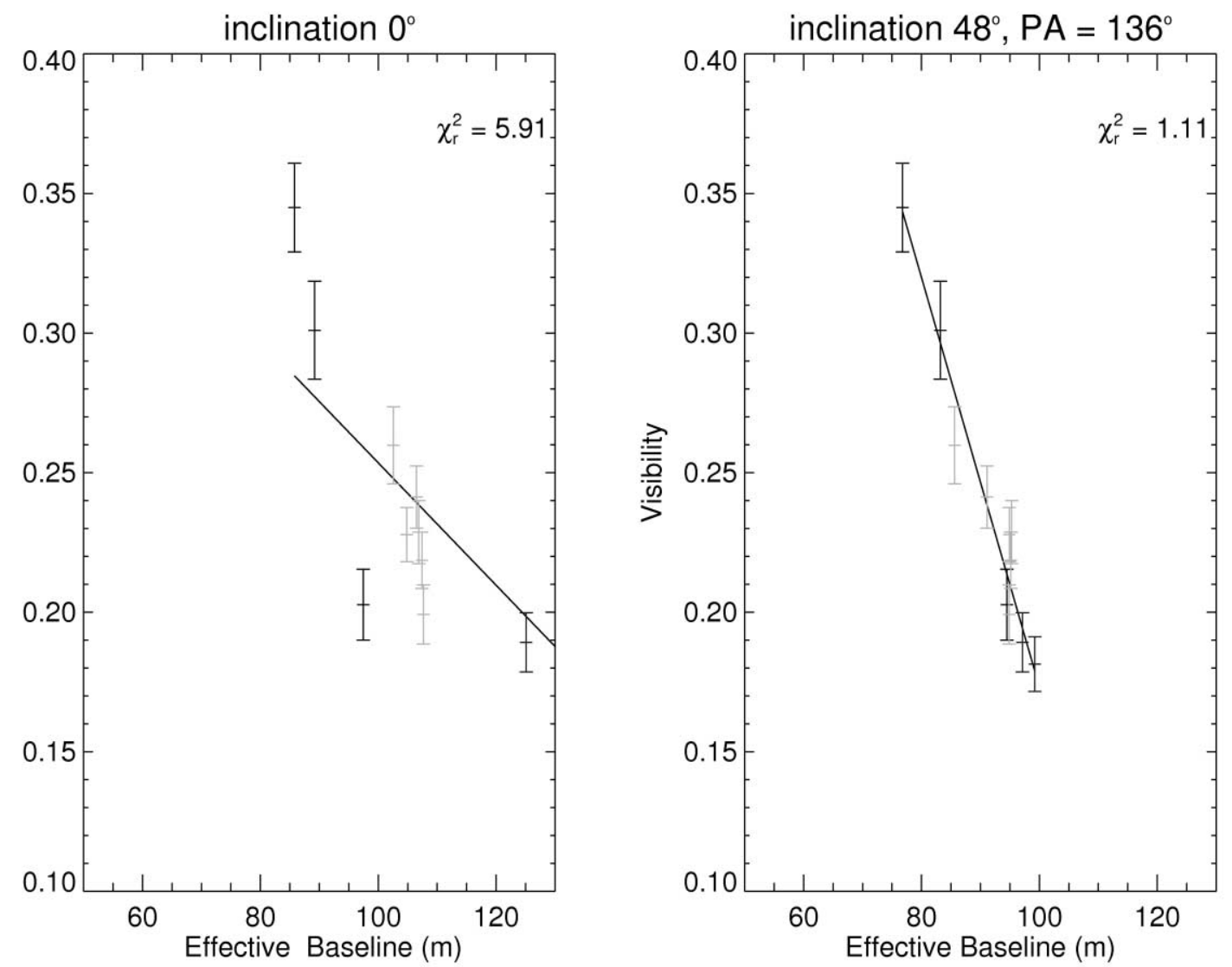

FIG. 20.-Linear fits to the observed visibilities (obtained with the W1W2, S2W2, and E2S2 CHARA telescope pairs) as a function of effective interferometric baseline. The data symbols are explained in Fig. 3. [See the electronic edition of the Journal for a color version of this figure.] 
Barth, A. J. 2001, ASP Conf. Ser. 238, Astronomical Data Analysis Software and Systems X, ed. F. R. Harnden, Jr., F. A. Primini, \& H. E. Payne (San Francisco: ASP), 385

Berger, D. H., Monnier, J. D., Millan-Gabet, R., ten Brummelaar, T. A., Muirhead, P., Pedretti, E., \& Thureau, N. 2006, Proc. SPIE, 6268, 62683K Calvet, N., Magris, G. C., Patino, A., \& D’Alessio, P. 1992, Rev. Mex. AA, 24, 27 Chiang, E. I., \& Goldreich, P. 1997, ApJ, 490, 368

Corder, S., Eisner, J., \& Sargent, A. 2005, ApJ, 622, L133

Dullemond, C. P., \& Dominik, C. 2004, A\&A, 421, 1075

Dullemond, C. P., Dominik, C., \& Natta, A. 2001, ApJ, 560, 957

Dullemond, C. P., \& Natta, A. 2003, A\&A, 408, 161

Eisner, J. A. 2007, Nature, 447, 562

Eisner, J. A., Chiang, E. I., Lane, B. F., \& Akeson, R. L. 2007, ApJ, 657, 347

Eisner, J. A., Lane, B. F., Akeson, R. L., Hillenbrand, L. A., \& Sargent, A. I. 2003, ApJ, 588, 360

Eisner, J. A., Lane, B. F., Hillenbrand, L. A., Akeson, R. L., \& Sargent, A. I. 2004, ApJ, 613, 1049

Ferguson, J. W., Alexander, D. R., Allard, F., Barman, T., Bodnarik, J. G., Hauschildt, P. H., Heffner-Wong, A., \& Tamanai, A. 2005, ApJ, 623, 585

Fukagawa, M., et al. 2004, ApJ, 605, L53

Garcia Lopez, R., Natta, A., Testi, L., \& Habart, E. 2006, A\&A, 459, 837

Grady, C. A., Woodgate, B., Bruhweiler, F. C., Boggess, A., Plait, P., Lindler, D. J., Clampin, M., \& Kalas, P. 1999, ApJ, 523, L151

Grady, C. A., et al. 2000, ApJ, 544, 895

Harries, T. J. 2000, MNRAS, 315, 722

Harries, T. J., Monnier, J. D., Symington, N. H., \& Kurosawa, R. 2004, MNRAS, 350,565

Hillenbrand, L. A., Strom, S. E., Vrba, F. J., \& Keene, J. 1992, ApJ, 397, 613

Isella, A., \& Natta, A. 2005, A\&A, 438, 899

Isella, A., Natta, A., \& Testi, L. 2007, A\&A, 469, 213

Isella, A., Testi, L., \& Natta, A. 2006, A\&A, 451, 951

Jones, B., \& Puetter, R. 1993, Proc. SPIE, 1946, 610

Kraus, A., Preibisch, T., \& Ohnaka, K. 2008, ApJ, 676, 490

Kurosawa, R., Harries, T. J., Bate, M. R., \& Symington, N. H. 2004, MNRAS, 351,1134

Kurucz, R. L. 1970, SAO Special Report 308 (Cambridge: SAO)

Landolt, A. U. 1983, AJ, 88, 439

Leinert, Ch., van Boekel, R., Waters, L. B. F. M., Chesneau, O., Malbet, F., \& Köhler, R. 2004, A\&A, 423, 537

Lin, S., Ohashi, N., Lim, J., Ho, P., Fukagawa, M., \& Tamura, M. 2006, ApJ, 645, 1297

Liu, W. M., Hinz, P. M., Meyer, M. R., Mamajek, E. E., Hoffmann, W. F., Brusa, G., Miller, D., \& Kenworthy, M. A. 2007, ApJ, 658, 1164

Lucy, L. B. 1999, A\&A, 344, 282

Mannings, V., \& Sargent, A. I. 1997, ApJ, 490, 792

Mariñas, N., Telesco, C. M., Fisher, R. S., Packham, C., \& Radomski, J. T. 2006, ApJ, 653, 1353
Meeus, G., Waters, L. B. F. M., Bouwman, J., van den Ancker, M. E., Waelkens, C., \& Malfait, K. 2001, A\&A, 365, 476

Merand, A., Borde, P., \& Coude Du Foresto, V. 2005, A\&A, 433, 1155

Millan-Gabet, R., Schloerb, F. P., \& Traub, W. A. 2001, ApJ, 546, 358

Millan-Gabet, R., Schloerb, F. P., Traub, W. A., Malbet, F., Berger, J. P., \& Bregman, J. D. 1999, ApJ, 513, L131

Miroshnichenko, A., Ivezic, Z., \& Elitzur, E. 1997, ApJ, 475, L41

Monnier, J. D., Tannirkulam, A., Tuthill, P. G., Ireland, M., Cohen, R., Danchi, W. C., \& Baron, F. 2008, ApJ, 681, L97

Monnier, J. D., Tuthill, P. G., Ireland, M. J., Cohen, R., \& Tannirkulam, A. 2004, BAAS, 36, 1367

Monnier, J. D., et al. 2007, Science, 317, 342 2006, ApJ, 647, 444 2005, ApJ, 624, 832

Muzerolle, J., D’Alessio, P., Calvet, N., \& Hartmann, L. 2004, ApJ, 617, 406

Natta, A., Prusti, T., Neri, R., Wooden, D., Grinin, V. P., \& Mannings, V. 2001, A\&A, 371, 186

Natta, A., Testi, L., Neri, R., Sheperd, D. S., \& Wilner, D. J. 2004, A\&A, 416, 179 Oppenheimer, B. R., et al. 2008, ApJ, 679, 1574

Ossenkopf, V., Henning, Th., Mathis, J. S. 1992, A\&A, 261, 567

Pinte, C., Menard, F., Berger, J. P., Benisty, M., \& Malbet, F. 2008, A\&A, in press

Pollack, J. B., Hollenbach, D., Beckwith, S., Simonelli, P. D., Roush, T., \& Fong, W. 1994, ApJ, 421, 615

Pontoppidan, K. M., Dullemond, C. P., Blake, G. A., Adwin Boogert, A. C., van Dishoeck, E. F., Evans, N. J., Kessler-Silacci, J., \& Lahuis, F. 2007, ApJ, 656,980

Semenov, D., Pavlyuchenkov, Ya., Schreyer, K., Henning, Th., Dullemond, C., \& Bacmann, A. 2005, ApJ, 621, 853

Shakura, N. I., \& Sunyaev, R. A. 1973, A\&A, 24, 337

Sitko, S. L., et al. 2008, ApJ, 678, 1070

Tannirkulam, A. T., Harries, T. J., \& Monnier, J. D. 2007, ApJ, 661, 374

Tannirkulam, A. T., et al. 2008, ApJ, 677, L51 (T08)

ten Brummelaar, T. A., et al. 2005, ApJ, 628, 453

Tuthill, P. G., Monnier, J. D., \& Danchi, W. C. 2001, Nature, 409, 1012

van Boekel, R., Dullemond, C. P., \& Dominik, C. 2005a, A\&A, 441, 563

van Boekel, R., Min, M., Waters, L. B. F. M., de Koter, A., Dominik, C., van den Ancker, M. E., \& Bouwman, J. 2005b, A\&A, 437, 189

Vinkovic, D., Ivezic, Z., Jurkic, T., \& Elitzur, M. 2006a, ApJ, 636, 348

Walker, C., Wood, K., Lada, C. J., Robitaille, T., Bjorkman, J. E., \& Whitney, B. 2004, MNRAS, 351, 607

Wassell, E. J., Grady, C. A., Woodgate, B., Kimble, R. A., \& Bruhweiler, F. C. 2006, ApJ, 650, 985

Weiner, J., et al. 2006, ApJ, 636, 1067

Wisniewski, J. P., Clampin, M., Grady, C. A, Ardila, D. R., Ford, H. C., Gomilowski, D. A., Illingworth, G. D., \& Krist, J. E. 2008, ApJ, 682, 548

Zhu, Z., Hartmann, L., Calvet, N., Hernandez, J., Muzerolle, J., \& Tannirkulam, A. 2007, ApJ, 669, 483 